\title{
Der Kampf um knappe Mittel: Die Bestimmungsfaktoren der öffentlichen, privaten und sektoralen Bildungsausgaben im OECD-Länder-Vergleich*
}

\author{
Marius R. Busemeyer
}

Dieser Aufsatz widmet sich der Bestimmung der Determinanten der öffentlichen, privaten und sektoralen Bildungsausgaben in 21 entwickelten OECD-Demokratien zwischen 1980 und 2001 unter Verwendung statistisch-quantitativer Methoden. Zunächst werden unter Zuhilfenahme der etablierten Theorien der international vergleichenden Staatstätigkeitsforschung Hypothesen entwickelt, die anschließend dem empirischen Test unterzogen werden. Dabei kann die Bedeutung von Programm- und Finanzierungskonkurrenzen zwischen den Bildungsausgaben und sozialkonsumtiven Ausgabenfeldern wie der Renten-oder Arbeitslosenpolitik nachgewiesen werden. Außerdem zeigt sich, dass ökonomische und demographische Variablen eine hohe Erklärungskraft haben. Aber auch parteipolitische und Machtressourcenvariablen sind nicht zu vernachlässigen. Die Globalisierungshypothese hat sich vor allem zur Erklärung der öffentlichen Tertiärausgaben als hilfreich erwiesen. Allgemein gesprochen kann ein gewisser Substitutionseffekt zwischen den öffentlichen und privaten Bildungsausgaben nachgewiesen werden. Eine ausgebaute konstitutionelle Vetostruktur (eine Vielzahl formaler Vetospieler), die sich nicht nur aufdie Sozial-, sondern auch auf die Entwicklung der Bildungsausgaben bremsend auswirkt, fübrt bei entsprechender demographischer Bildungsnachfrage zu hohen Privatausgaben. Besonderer Bedarf nach weiterer Forschung ist bei der näheren Erkundung des positiven Zusammenhangs zwischen der Frauenerwerbsquote und den öffentlichen, privaten und tertiären Bildungsausgaben anzumelden.

\footnotetext{
* Die Forschungsarbeiten zu diesem Projekt wurden im Rahmen des DFG-Projektes „Bildungsausgaben im internationalen Vergleich" unter Leitung von Prof. Manfred G. Schmidt am Institut für Politische Wissenschaft der Universität Heidelberg durchgeführt. Ich möchte Manfred Schmidt, Uwe Wagschal, Rita Nikolai, Frieder Wolf, Nico Siegel, Frank Castles, den Teilnehmern des Heidelberger Doktoranden- und Habilitandenkolloquiums sowie den zwei anonymen Gutachtern der PVS für hilfreiche Anmerkungen und Kritik danken. Sämtliche verbliebenen Fehler gehen selbstverständlich auf meine Verantwortung.
} 


\section{Einleitung und Fragestellung}

Dieser Aufsatz beschäftigt sich mit der statistisch-quantitativen Analyse der Bestimmungsfaktoren der Bildungsausgaben im Vergleich der 21 wirtschaftlich entwickelten OECD-Länder. ${ }^{1}$ Dabei sollen sowohl die privaten, die öffentlichen als auch die nach Bildungssektoren gegliederten Ausgaben im Mittelpunkt der Analyse stehen. Unter Verwendung von vor allem aus der Sozialausgaben- und Staatsausgabenforschung bekannten Theorien werden Hypothesen zum Politikfeld Bildung abgeleitet, die anschließend in einer statistischen Untersuchung dem empirischen Test unterworfen werden. Der politische Kampf um die zunehmend knapper werdenden öffentlichen Mittel ist ein roter Leitfaden bei dieser Untersuchung. Wie deutlich werden wird, stehen die Bildungs- und die Sozialpolitik in einer Finanzierungskonkurrenz (Lepenies 2003) zu einander. Dies gilt für manche Länder stärker als für andere und ist abhängig von der jeweiligen institutionellen Struktur des Wohlfahrtsstaates.

Die Analyse der Bildungspolitik ist bislang nicht nur, aber vor allem von der deutschen Politikwissenschaft vernachlässigt worden (Reuter 2002). Das fällt vor allem dann auf, wenn man auf den inzwischen beträchtlichen Stand der Forschung in Nachbardisziplinen wie der Ökonomie (Timmermann 2002) und der Soziologie (Allmendinger/Aisenbrey 2002) blickt. Es gibt eine Reihe von deskriptiv-vergleichenden Studien, die meist aus der Perspektive eines Landes geschrieben sind (Färber 2000; Weiß 1999; Hetmeier/Weiß 2001; Klemm 2003 (alle aus der Perspektive Deutschlands); Glennerster 2001 (UK); Nelson 1992, 1996 (USA); Heynemann 2001; O’Higgins 1988 (globale Perspektive)). Ein zweiter Literaturstrang verwendet zur Erklärung der Variation der Bildungsausgaben vor allem sozioökonomische und weniger politisch-institutionelle Variablen. Beispiele für diese Arten von Studien, bei denen unter anderem das Pro-Kopf-Einkommen, die Bildungspartizipation ${ }^{2}$ oder die Höhe der Studiengebühren als Determinanten der Bildungsausgaben identifiziert werden, sind Hanushek/ Rivkin (1996), Fernandéz/Rogerson (1997), Morgan et al. (2001) und Ram (1995).

Die dritte Kategorie der Studien, die sich mit der Erforschung der Bildungsausgaben beschäftigt haben, beinhaltet Arbeiten, die sowohl sozioökonomische „sources“ als auch politisch-institutionelle "causes" berücksichtigen. ${ }^{3}$ Ein frühes Beispiel ist die Studie von Cameron und Hofferbert (1974), die auch den Zusammenhang zwischen Bildungsausgaben und Föderalismus betrachtet. Verner (1979) legte einen Most-Dissimilar-Cases-Vergleich zur Untersuchung der Bildungsausgaben in 102 Ländern vor. Besonders zu würdigen sind außerdem die Arbeiten von Castles, der sich direkt (Castles 1989, 1998) und indirekt (Castles 1982; Castles/Marceau 1989; Castles/McKinlay 1979) mit der Untersuchung der Bildungsausgaben beschäftigt hat. Er hebt die Bedeutung des Faktors Religion, aber auch die Wichtigkeit parteipolitischer Variablen hervor.

1 Australien, Belgien, Kanada, Dänemark, Finnland, Frankreich, Deutschland, Griechenland, Italien, Irland, Japan, Neuseeland, Niederlande, Norwegen, Österreich, Portugal, Spanien, Schweden, Schweiz, Großbritannien und die USA.

2 Prinzipiell ist darunter der Anteil der Schüler bzw. Studenten an der jeweils relevanten Altersgruppe zu verstehen.

3 Vgl. zur Unterscheidung zwischen den oberflächlich wirkenden „sources“ und den tiefer liegenden "causes" Olson (1982: 4). 
Die Untersuchung des Zusammenhangs zwischen Bildungsausgaben und der parteipolitischen Zusammensetzung der Regierung ist von einigen fortgeführt worden (Garrett/Lange 1991; Boix 1997, 1998). Hokenmaier (2002) und Hega/Hokenmaier (2002) verwenden das Castles-Modell als Ausgangspunkt für eigene Untersuchungen der Bildungsausgaben. Andere beschäftigen sich mit der Analyse der Wirkung einzelner unabhängiger Variablen wie der Globalisierung (Kaufman/Segura-Ubiergo 2001), der demographischen Zusammensetzung der Bevölkerung (Poterba 1997) oder dem Einfluss direktdemokratischer Entscheidungsverfahren (Santerre 1989; Sass 1991). In jüngerer Zeit hat sich vor allem Manfred G. Schmidt eindringlich mit der Analyse der Bestimmungsfaktoren der Bildungsausgaben beschäftigt (Schmidt 2002b, 2003a, 2004).

Der vorliegende Aufsatz möchte Lücken im bestehenden Stand der Forschung schließen. Diese Lücken sind vor allem in drei Punkten zu sehen: Erstens ist die Perspektive der meisten der vorliegenden Studien beschränkt auf die deskriptive Beschreibung, den einfachen Querschnitt- oder den einfachen Längsschnittvergleich. Eine Kombination der Querschnitt- und der Längsschnittperspektive mit Hilfe der Methode der gepoolten Zeitserienanalyse soll eine umfassende Betrachtung der Bestimmungsfaktoren der Ausgaben ermöglichen, die beide Perspektiven miteinander verbindet. Zweitens beschäftigt sich ein Großteil der vorliegenden Studien mit der Analyse der öffentlichen Ausgaben (Castles 1989, 1998; Hokenmaier 2002). Die vorliegenden Daten aus Quellen der OECD (Education at a Glance-Reihe) ermöglichen jedoch bis zu einem gewissen Grad die Analyse der privaten Ausgaben (den Arbeiten von Schmidt (2003a, 2004) folgend) sowie die Aufteilung auf die einzelnen Bildungssektoren (Primär- und Sekundärbereich auf der einen Seite sowie Hochschulsektor andererseits). Die Breite der Analyseperspektive dieser Arbeit soll daher über die der meisten bisherigen Studien hinausreichen. Drittens geht es um eine umfassende Analyse auch im Hinblick auf die betrachteten Variablen. Wie in dem kurzen Überblick über die relevante Literatur deutlich geworden sein dürfte, betrachten viele Arbeiten, vor allem diejenigen mit wirtschaftswissenschaftlichem Hintergrund, lediglich die kurzfristig wirkenden sozioökonomischen Variablen und vernachlässigen längerfristig wirkende politische und institutionelle Parameter. Auch dieser Missstand soll durch die Anwendung der Theorien der international vergleichenden Staatstätigkeitsforschung (Schmidt 2001; Castles 2002) in dieser Arbeit gelindert werden.

\section{Theorie und Hypothesen}

Die untersuchten abhängigen Variablen in dieser Studie sind die öffentlichen, privaten und sektoralen Bildungsausgaben in Prozent des Bruttoinlandsproduktes (BIP) in 21 OECD Staaten im Zeitraum zwischen 1980 und 2001. Die betrachteten unabhängigen Variablen und die sie betreffenden Hypothesen werden im Folgenden entlang des aus der Sozialpolitik- und Staatsausgabenanalyse bekannten Katalogs der Theorien der international vergleichenden Staatstätigkeitsforschung vorgestellt (Schmidt 1993, 2001; Castles 2002; Siegel 2002). 


\subsection{Die sozioökonomische Schule}

Die Theorien der sozioökonomischen Schule rücken Variablen zum wirtschaftlichen und demographischen Hintergrund wie wirtschaftlichen Wohlstand, Wirtschaftswachstum und die demographische Zusammensetzung der Bevölkerung in den Mittelpunkt der Betrachtung (Zöllner 1963; Wilensky 1975, 2002). Im Falle der Sozialpolitik besteht eine relativ enge Verbindung zwischen den Ausgaben und dem Wirtschaftswachstum. Hohe Arbeitslosenzahlen erhöhen die Zahl der Leistungsempfänger und belasten die Sozialkassen. Bei der Bildungspolitik ist der Zusammenhang allerdings eher indirekter Natur. Primär- und Sekundärbildung hängen weniger stark von kurzfristigen Schwankungen der wirtschaftlichen Konjunktur ab, weil es um die konstante Bereitstellung einer bildungspolitischen Grundversorgung geht. Ausgaben für post-sekundäre Bildung könnten jedoch stärker von ökonomischen Bedingungen abhängig sein.

Der Stand der wirtschaftichen Entwicklung, gemessen am BIP pro Kopf, ist eine weitere, in der sozioökonomischen Schule beliebte Variable. Das Wagnersche Gesetz (Schmidt 1998: 161), vom Nationalökonomen Adolph Wagner zur Analyse der Sozialausgaben geprägt, geht dabei von einem positiven Zusammenhang zwischen dem wirtschaftlichen Entwicklungsstand und der öffentlichen Ausgabenquote (Staatsquote) aus, d.h. je reicher ein Land, desto mehr, relativ zur Wirtschaftskraft, gibt es für Soziales, Bildung und andere öffentliche Güter aus. Im Unterschied zu den Sozialausgaben, die bis spät ins 20. Jahrhundert hinein eine starke Wachstumsdynamik entfaltet haben, haben sich die Bildungsausgabenquoten in den entwickelten Industrienationen schon relativ früh stabilisiert. Der Wachstumstrend war hier insgesamt auch schwächer ausgeprägt. Die Bildungsexpansion hat sich, nachdem eine universale Primär- und Sekundärbildung zum integralen Bestandteil des Konzeptes des modernen, westlich geprägten Nationalstaates geworden war (Meyer et al. 1992), nach dem Zweiten Weltkrieg vor allem im post-sekundären Sektor abgespielt. Starkes Wirtschaftswachstum in der ersten Nachkriegszeit konnte hier für die Bereitstellung der notwendigen Ressourcen sorgen, ohne dass der Anteil der Bildungsausgaben am BIP insgesamt stark gewachsen wäre. Castles (1989: 441-442) kann in seinen Analysen die Gültigkeit des Wagnerschen Gesetzes bei den Bildungsausgaben nicht nachweisen. Dies würde darauf hindeuten, dass die Bildungsausgaben schon relativ früh an diese "Sättigungsgrenze" gestoßen sind. Eine positive Assoziation allerdings wäre Beleg dafür, dass das Wachstumspotenzial der post-sekundären Bildungsexpansion, von der angenommen werden kann, dass sie in den wirtschaftlich entwickelten Staaten schneller voranschritt, durch die Erschließung neuer Nachfragegruppen (wirtschaftlich Schwache, Frauen) so groß ist, dass auch der relative Anteil der Bildungsausgaben am BIP ansteigt.

Das demographische Umfeld spielt sicherlich als Determinante der Bildungsausgaben eine ungemein wichtige Rolle. Die Demographie definiert „latente Gruppen“ (Olson 1992), die zu einem gewissen Grad durch objektiv zuschreibbare, gemeinsame Interessen bestimmt werden. In diesem Zusammenhang ist es sehr hilfreich, den politischen Prozess nicht aus der herkömmlichen Perspektive als politischen Kampf zwischen Interessengruppen, Parteien, Gewerkschaften, Arbeitgeberverbänden und anderen bekannten politischen Akteuren zu begreifen. Vielmehr macht die Perspektive der "generational politics" (Heclo 1988) darauf aufmerksam, dass politische Konflikte auch zwischen 
Generationen ausgetragen werden können. Im Zuge der in der OECD-Welt zu beobachtenden Bevölkerungsalterung beschränken die Leistungsansprüche der Bevölkerungsgruppe der Rentner in zunehmendem Maße den fiskalischen Spielraum der öffentlichen Sozialversicherungssysteme (Pampel/Williamson 1988, 1989). Die Gruppe der Älteren wird außerdem zu einer wahlpolitisch entscheidenden und mächtigen, stillen Vetomacht (Pecchenino/Utendorf 1999), die die Sicherung der gewährten Ansprüche vor die aktive Zukunftsvorsorge stellt (Kielmansegg 2001). Die junge Generation der Schüler und Studenten, die vor allem in den Genuss von Bildungsleistungen kommen sollen, sind politisch schwach. Sie sind größtenteils nicht wahlberechtigt und daher auf die stellvertretende Wahrnehmung ihrer Interessen durch die Eltern angewiesen. Diese haben zwar ein gewisses Interesse an der Aufrechterhaltung eines Mindestmaßes an Humankapitalinvestitionen zur Sicherstellung der langfristigen ökonomischen Grundlagen des Systems und zur Sicherung ihrer zukünftigen Rentenansprüche. Die stellvertretende Wahrnehmung der Interessen der Jungen kann allerdings schnell in Konflikt kommen mit anderen, konkreten Bedürfnissen der erwerbstätigen Generationen, wie zum Beispiel die Großzügigkeit der Leistungen aus Arbeitslosen- und Krankenversicherung. Eine positive Assoziation zwischen dem Bevölkerungsanteil der 5- bis 29-Jährigen und den Bildungsausgabenquoten würde überpessimistischen Hypothesen aus der Schule der "generational politics" widerlegen, denn es könnte nachgewiesen werden, dass Staaten mit einer relativ jungen Bevölkerung auch relativ mehr für Bildung ausgeben. ${ }^{4}$

\subsection{Institutionelle Faktoren}

Im Rahmen einer statistisch-quantitativen Analyse kommt institutionellen Faktoren vor allem in der Querschnittdimension eine hohe Erklärungskraft zu. Die verfassungs- und gewohnheitsrechtlich kodifizierte Stellung der zentralstaatlichen Regierung, die durch den Grad der Ausprägung der konstitutionellen Vetostruktur (Czada 2003: 176-177) bzw. die Zahl der institutionalisierten Mehrheitsbegrenzer wesentlich bestimmt wird, ist eine aus der Sozialausgabenforschung bekannte Variable (Hicks/Swank 1992; Huber et al. 1993; Schmidt 2000: 352-353; Huber/Stephens 2001a, 2001b; Kittel/Obinger 2003: 30). Föderalismus, Bikameralismus, ein starkes Verfassungsgericht, eine unabhängige Zentralbank, Direktdemokratie oder EU-Mitgliedschaft - dies sind die institutionalisierten Vetopositionen, die von in diesen Theorien gebräuchlichen Makro-Indizes erfasst werden. Aus der Sozialausgabenforschung ist bekannt, dass eine ausgeprägte Vetostruktur die Expansion des Wohlfahrtsstaates tendenziell gebremst (Obinger/Wagschal 2000; Schmidt 2002a: 181-182) und umgekehrt die Konzentration von politischer Macht in den Händen der Zentralregierung die Expansion beschleunigt hat (Hicks/Swank 1992: 661-662). Diese Zusammenhänge können dadurch erklärt werden, dass in Staaten, in denen der Zentralregierung eine große Steuerungsmacht zukommt, die Expansion der öffentlichen Ausgaben nicht durch Vetospieler wie regionale

4 Die kausale Richtung dieses Zusammenhangs ist allerdings nicht hundertprozentig klar: Eine positive Assoziation zwischen den beiden Variablen könnte auch belegen, dass es den Staaten die viel in Bildung investieren, gelingt, dadurch hohe Geburtenraten und daher eine relativ junge Bevölkerung zu bewirken. 
Gebietskörperschaften, Direktdemokratie oder eine starke Verfassungsgerichtsbarkeit gebremst wurde.

Eine ausgeprägte konstitutionelle Vetostruktur ist dann negativ mit der Höhe der öffentlichen Bildungsausgaben assoziiert, wenn der Bremseffekt der Vetopunkte nicht nur die Sozialausgaben, sondern auch die generelle Arbeitsteilung zwischen Staat und Markt in der Bereitstellung öffentlicher Güter betrifft und Bildungsausgaben als Teil eines umfassenden wohlfahrtsstaatlichen Arrangements angesehen werden. In Bezug auf die privaten Bildungsausgaben müsste demnach eine andere Beziehung gelten: Wenn eine ausgebaute Vetostruktur die öffentlichen Ausgaben im Zaum hält, dann ist zu erwarten, dass durch eine höhere Beteiligung der privaten Hand ein Teil der Bildungsnachfrage befriedigt wird, wenn eine entsprechende, demographisch bedingte Nachfrage besteht (hoher Anteil der 5- bis 29-Jährigen).

Ökonomische Föderalismustheorien (Kirchgässner 2001; Kirchgässner/Pommerehne 1997) gehen bei der Variablen Föderalismus generell von einem ausgabenmindernden Effekt aus. Dies könne zurückgeführt werden auf den Wettbewerb zwischen den regionalen Gebietskörperschaften (Wettbewerbsthese), die sich in ihren Steuerforderungen gegenseitig unterbieten, oder auf die bessere Anbindung der politischen Klasse an die lokalen Präferenzen (Dezentralisierungsthese). Eine genauere Betrachtung der föderalistischen Länder fördert allerdings zu Tage, dass es auch enorme Unterschiede gibt zwischen diesen Ländern hinsichtlich der tatsächlichen fiskalischen Autonomie, die den unteren Regierungsebenen eingeräumt wird (Braun 2000). Klassisch ist die Unterscheidung zwischen Systemen des Trenn- (USA) und Verbundföderalismus (Deutschland). Aber auch über diese Unterscheidung hinaus ist die Erfassung der fiskalischen lokalen Autonomie bedeutsam. Die Betrachtung von Ausgabenanteilen der einzelnen Regierungsebenen reicht dabei nicht aus (Stegarescu 2004: 1), da Ausgaben auf der lokalen Ebene von oben mandatiert sein können. Insofern ist vor allem die Frage in den Mittelpunkt zu stellen, ob lokale Einheiten steuerpolitisch relevante Entscheidungen (z.B. über Steuersätze und Bemessungsgrundlagen) selbst treffen können und ob sie Steuereinnahmen selbst erheben und verwalten (Stegarescu 2004: 6). Stegarescus Indikatoren zeigen, dass die tatsächliche fiskalische Autonomie in konstitutionell föderalistischen Staaten wie Deutschland und Österreich geringer ist als in konstitutionell unitarischen Staaten wie in Skandinavien, den Niederlanden oder Großbritannien.

In Bezug auf die Bildungsausgaben ist davon auszugehen, dass sich konstitutionell föderalistische von unitarischen Staaten nicht per se in der Gesamthöhe der Ausgaben unterscheiden, auch wenn sich Unterschiede hinsichtlich der innerstaatlichen Variationsbreite ergeben (Cameron/Hofferbert 1974). Eine hohe fiskalische Autonomie der lokalen Gebietskörperschaften könnte jedoch einen positiven Effekt auf die Gesamthöhe der Ausgaben haben, wie Landon (1999) im Fall Kanada nachweisen konnte. Lokale Einheiten befinden sich bei der Bildungspolitik nicht in einem „race to the bottom", sondern vielmehr in einem Überbietungswettbewerb: Auf lokaler Ebene stehen den Individuen reale Exit-Optionen zur Verfügung, und ein Umzug von einer Lokalität in die andere, weil es dort bessere Schulen gibt, ist eine reale Möglichkeit. Wenn die Lokalitäten frei über ihre Steuern verfügen können, ist es für sie rational, unter Ausnutzung der "fiscal illusion" dem Wähler möglichst gute Schulen zu bieten, auch wenn dieser sich nicht immer der Tatsache bewusst ist, dass er durch seine Steuern 
letzten Endes dafür bezahlen muss. Insofern ist zwischen den Indikatoren der fiskalischen Dezentralisierung und den Bildungsausgaben von einem positiven Zusammenhang auszugehen.

Von besonderer Bedeutung in diesem Aufsatz sind Variablen, die den Grad der Programm- und Finanzierungskonkurrenz zwischen der Bildungspolitik und anderen Ausgabenfeldern messen. Ein Großteil der Ausgabenforschung verfügt über die Schwäche, dass sie die Wechselbeziehungen zwischen Politik- und Ausgabenfeldern zugunsten der isolierten Betrachtung eines einzelnen Ausgabenbereiches vernachlässigt. Daher soll in dieser Arbeit das Verhältnis der Finanzierungskonkurrenz (Lepenies 2003) zwischen Bildungs- und Sozialpolitik eine wichtige Rolle spielen. ${ }^{5}$ Dabei ist die Unterscheidung zwischen der Querschnitt- und der Längsschnittdimension von enormer Bedeutung. Im Querschnitt betrachtet, zeigt sich im Vergleich der westlichen Wohlfahrtsstaaten ein eigentümliches Ausgabenmuster, das die in den einzelnen Welten der Wohlfahrtsstaatlichkeit (Esping-Andersen 1990) vorherrschenden sozial- und bildungspolitischen Prioritäten eindrücklich widerspiegelt. Die kontinentaleuropäischen, so genannten konservativen Wohlfahrtsstaaten geben im Vergleich zu den restlichen OECD-Demokratien relativ wenig für Bildung, aber umso mehr für transferintensive, kompensierende Sozialpolitik aus. In der liberalen, vornehmlich angelsächsischen Welt können wir nahezu ein Spiegelbild dieses Musters identifizieren: Relativ hohe Ausgaben für Bildung, besonders wenn man sowohl öffentliche als auch private Quellen berücksichtigt, aber relativ niedrige Ausgaben für Sozialpolitik. Die Staaten der sozialdemokratischen, vornehmlich skandinavischen Welt hingegen sind sowohl bei den Sozial- als auch bei den Bildungsausgaben internationale Spitzenreiter (Kohl 1981; Hega/Hokenmaier 2002; Hokenmaier 2002; Allmendinger/Leibfried 2003; Leibfried 2003). Diese Ausgabenmuster geben den Stellenwert der Bildung (Chancengleichheit) gegenüber der primär kompensierenden und ausgleichen Sozialpolitik (Gleichheit der Outcomes) wieder. Der Stellenwert der Bildung ist somit vor allem in den Staaten gering, in denen die öffentlichen finanziellen Ressourcen durch aufwändige öffentliche Sozialversicherungssysteme für Rente und bzw. oder Arbeitslosigkeit stark belastet werden. In den universalistischen Sozialstaaten Skandinaviens hingegen ist der Wohlfahrtsstaat durch die aktive Förderung von Familienpolitik sowie die ausgeprägten Bemühungen zur Beschäftigungsförderung von Frauen und anderen ehemals vom Arbeitsmarkt ausgeschlossenen Bevölkerungsgruppen der Bildung insgesamt positiver gesinnt. Eine wichtige Variable hierbei ist außerdem die Zahlungsbereitschaft des Wahlvolkes, die zum Teil auch durch Regierungshandeln beeinflusst werden kann.

Andere Auswirkungen der Finanzierungskonkurrenz zwischen sozialpolitischen Ausgabenfeldern zeigen sich vor allem in der Längsschnittdimension. Mit dem Anbrechen der Ära der „New Politics of the Welfare State" (Pierson 1994, 1996, 2001) und dem sie begleitenden fiskalischen Klima der Knappheit und Austerität hat sich die Konkurrenz der Ausgabenbereiche um die knappen öffentlichen Mittel weiter verschärft. Die

\footnotetext{
5 An dieser Stelle ist es wichtig zu betonen, dass die im Rahmen einer quantitativen Analyse ermittelten Assoziationen zwischen verschiedenen Ausgabenfeldern eine andere Aussagequalität hinsichtlich der Kausalität haben als dies bei anderen unabhängigen Variablen der Fall ist. Aus gabenparameter, auch wenn sie unterschiedliche Politikfelder betreffen, hängen oft kausal von den gleichen Bestimmungsfaktoren ab (politisch-institutionell, sozioökonomisch).
} 
Bildungspolitik zieht dabei, zumindest in einigen Staaten, den Kürzeren. Der Erhalt der sozialpolitischen Errungenschaften wird durch mächtige Interessengruppen wie Gewerkschaften oder Sozialverbände sowie autonome Sozialversicherungsinstitutionen verteidigt. Die Größe und politische Macht der Sozialstaatsklientel von Arbeitlosen und Sozialhilfeempfängern über Rentner bis hin zu den Leistungsempfängern der öffentlichen Krankenversicherungen ist beträchtlich. Zwar ist auch die Größe der Bildungsklientel und ihrer Abhängigen (Lehrer, Schüler, Eltern, Studenten) nicht unbeträchtlich. Im Unterschied zu den Sozialstaatsklientelgruppen verfügen sie aber nicht nur über ein geringeres Organisationspotenzial, sondern auch über ein geringeres Konfliktpotenzial (Offe 1973: 25), d.h. sie können zur Durchsetzung ihrer Ansprüche kaum mit dem Entzug von für die Gesellschaft lebenswichtigen Ressourcen drohen. ${ }^{6}$ Eine hohe Staatsverschuldung, gemessen am gesamten Schuldenstand oder der Zinslastquote (Zinszahlung in Prozent des BIP), intensiviert den Kampf um knappe Mittel. Wenn die These, dass die Bildungsausgaben diesen Kampf verlieren, stimmt, dann müsste sich zwischen den Indikatoren der Staatsverschuldung und den Ausgaben eine negative Assoziation nachweisen lassen.

\subsection{Parteiendifferenzlehre}

Die These, dass die parteipolitische Zusammensetzung der Regierung auch im Policy-Output und Outcome einen Unterschied macht, ist vor allem seit Anfang der 1980er Jahre in der Sozialausgaben- und Staatstätigkeitsforschung populär geworden (Castles 1982; Schmidt 1982; Hibbs 1977, 1987; Cameron 1984, 1985). Die Standardthese ist dabei, dass linke Regierungen insgesamt ausgabenfreudiger sind als bürgerliche (Cameron 1978, 1985: 237; Esping-Andersen 1985: 235; Kohl 1981: 324; Schmidt 1982; Castles 1982: 71). Aber auch die Stärke und der Grad der Zersplitterung der Oppositionsparteien spielen eine Rolle (Hicks/Swank 1992). In Ländern mit zwei Sozialstaatsparteien (wie Deutschland) lassen sich bürgerliche Regierungen zudem von ihren Parteikonkurrenten "anstecken" und geben mehr aus als ihre konservativen Kollegen in anderen Ländern (Huber/Stephens 2001a: 313; Wilensky 2002: 239; Schmidt 1998: 168). In jüngerer Zeit lässt sich ein Abnehmen der Erklärungskraft der parteipolitischen Variablen beobachten (Huber/Stephens 2001a: 212; Kittel/Obinger 2003: 35; Stephens et al. 1999: 184), was zum Teil durch die aufgrund der fiskalischen Austerität erzeugte generelle Abnahme des fiskalpolitischen Handlungsspielraums erklärt werden kann.

In Bezug auf die Bildungsausgaben lässt sich ebenfalls die These aufstellen, dass linke (bürgerliche) Regierungen mehr (weniger) ausgeben für Bildung (Schmidt 2002b: 13; Castles 1998: 180; 1989: 441). Prinzipiell ist aber davon auszugehen, dass Klassengrenzen in der Bildungspolitik eine weniger ausgeprägte Rolle spielen als beispielsweise in der Wirtschaftspolitik (Schmidt 2003: 11). Die Wahlklientel linker Parteien, d.h. die unteren Einkommensschichten und die abhängig beschäftigten Arbeitnehmer, haben ein Interesse am Ausbau öffentlicher Bildungsmöglichkeiten, welche für sie und

6 Studentenstreiks mögen für Schlagzeilen sorgen, sind aber ansonsten weitgehend ineffektiv. Verbeamtete Lehrer dürfen nicht streiken. Schüler verfügen nicht über das notwendige politische Bewusstsein und die entsprechenden Organisationsfähigkeiten. Eltern nehmen die Interessen ihrer Kinder nur stellvertretend wahr. 
ihre Kinder die soziale Aufwärtsmobilität absichern. ${ }^{7}$ Die Unterstützergruppen bürgerlicher Parteien, die oberen Einkommensschichten, haben ein stärkeres Interesse daran, ihren Steuerbeitrag zu minimieren. Sie können es sich zudem besser leisten, Bildung über private Ausgaben zu finanzieren. Bei den Mitteparteien (vor allem Christdemokraten und Liberale) ist eine weniger starke Ausprägung der Parteiendifferenz zu erwarten.

\subsection{Machtressourcen}

Die Franenerwerbsquote ist eine bedeutsame Variable. Hier ist zu erwarten, dass eine starke positive Assoziation zu den Ausgaben besteht. Allerdings ist deren kausale Richtung weitgehend unklar. Ein ausgebauter Bildungsstaat gewährt Frauen vor allem durch den tertiären Sektor verbesserte Zugangschancen zum Arbeitsmarkt. Verstärkte Investitionen in die frühkindliche Erziehung und die Vorschulbildung sowie die Verfügbarkeit von finanzierbaren Kinderbetreuungsmöglichkeiten begünstigen ebenfalls die Frauenerwerbsbeteiligung. Allerdings ist, wie wir aus den skandinavischen Ländern wissen, der Bildungssektor für einen nicht unbeträchtlichen Teil der Frauen ein wichtiger Arbeitgeber. Insofern ist von einer kausalen Wechselbeziehung zwischen diesen beiden Variablen auszugehen, die so im Rahmen einer statistischen Analyse nicht nachweisbar sein kann: Hohe öffentliche Ausgaben für Bildung haben Frauen die Möglichkeit gegeben, sich notwendige Qualifikationen anzueignen. Eine hohe Frauenerwerbsquote kann dann wiederum die Ausgabenhöhe für Bildung in die Höhe treiben, wenn ein Großteil der neuen Jobs im Bildungsdienstleistungssektor angesiedelt ist. ${ }^{8}$

Abschließend spielt vor allem als Bestimmungsfaktor der privaten Bildungsausgaben der Anteil der im industriellen Sektor Beschäftigten an der Gesamtbeschäftigung als Indikator für die Bedeutung der industriellen Beschäftigung eine wichtige Rolle. Die privaten Bildungsausgaben in OECD-Staaten sind im Wesentlichen neben den Aufwendungen für private Schulen im Primär- und Sekundärbereich sowie für private Universitäten und Hochschulen die Ausgaben für berufliche Bildung auf der betrieblichen oder überbetrieblichen Ebene (post-sekundärer, nicht-tertiärer Sektor). Von daher sind die privaten Ausgaben in den Ländern hoch, die über ein teils privat, teils öffentlich finanziertes duales System der Berufsausbildung oder eine rein betriebliche Ausbildung verfügen (Schmidt 2004). Insofern ist auch zwischen dem Anteil der im industriellen Sektor Beschäftigten und den privaten Ausgaben (im Aggregat) eine positive Beziehung zu erwarten.

7 Im Fall der USA wird abweichend von dieser Hypothese die Existenz einer potenziellen Koalition der „ends against the middle“, also der reichen und armen Schichten, die beide ein Interesse an geringeren Bildungsausgaben haben (wenn auch aus verschiedenen Gründen), gegen die Mittelschicht, diskutiert. Die genaue empirische Überprüfung zeigt aber, dass es anstelle dieses U-förmigen einen positiven Zusammenhang gibt zwischen dem Medianeinkommen und den Präferenzen für hohe Bildungsausgaben (vgl. zu dieser Diskussion Cohen-Zada/Justman 2003; Ansell 2006).

8 Weil hier die Grenzen der multivariaten statistischen Methode erreicht sind, ist weitere Forschung besonders an diesem Punkt von hohem Nutzen. 


\subsection{Globalisierung: Die internationale Hypothese}

Von den Globalisierungstheoretikern werden zwei gegensätzliche Thesen über den $\mathrm{Zu}$ sammenhang zwischen Internationalisierung und Staatsausgaben in Stellung gebracht: Die Effizienzthese geht von einem ausgabenmindernden Einfluss aus, da der Wettbewerb zwischen den Nationen um das mobile Kapital den Steuerwettbewerb zwischen Staaten anheize und dadurch den fiskalpolitischen Spielraum nachhaltig begrenze (Garrett 2001: 6; Kaufman/Segura-Ubiergo 2001: 556). Die Kompensationsthese hingegen geht davon aus, dass besonders kleine und offene Volkswirtschaften, die gezwungen sind, die Bedingungen des Weltmarktes so zu akzeptieren, wie sie sind, auf einen ausgebauten Wohlfahrtsstaat angewiesen sind, da nur so die durch den verschärften ökonomischen Wettbewerb erzeugten sozialen Zerwürfnisse in den Griff zu bekommen sind (Cameron 1978; Rodrik 1997). Linksdominierte Regierungen haben, seitdem der Keynesianismus als wirtschaftspolitischer Ansatz sich als nicht mehr durchsetzungsund durchführungsfähig erwiesen hat, öffentliche Investitionen in das Humankapital als Instrument zur Stärkung der Wettbewerbsfähigkeit erkannt (Boix 1997). Wenn diese These zutrifft, ist besonders zwischen den öffentlichen Ausgaben für das post-sekundäre Bildungswesen und Globalisierungsindikatoren (zur Offenheit der Volkswirtschaft) eine positive Assoziation zu erwarten.

\subsection{Politikerbe}

Richard Rose (Rose 1990; Rose/Davies 1994) und andere (Wildavsky 1964) haben auf die enormen Trägheitsmomente staatlicher Ausgabenpolitik hingewiesen. Wenn nichts anderes über die Staatsfinanzen bekannt wäre, so würde die Ausgabenhöhe der Vorperiode dennoch einen relativ guten und genauen Schätzwert der aktuellen Ausgabengrößen liefern. Das vom Vorgänger hinterlassene Politikerbe prägt damit zu einem großen Maß auch die heutige Situation. Das Politikerbe bestimmt jedoch nicht nur das Niveau der heutigen Ausgaben, sondern auch dessen Veränderungsrate. Insbesondere ist hier ein gewisser Nachzüglereffekt (Catch up) zu erwarten, d.h. Staaten, die zu früheren Zeiten wenig für Bildung ausgegeben haben, haben in der Folgeperiode höhere Wachstumsraten zu verzeichnen als diejenigen, die bereits recht früh ein hohes Ausgabenniveau erreicht haben.

\section{Quantitative Analyse}

\subsection{Daten und Methoden}

Nachdem im zweiten Abschnitt unter Zuhilfenahme der etablierten Theorien der international vergleichenden Staatstätigkeitsforschung entsprechende Hypothesen zu den Bestimmungsfaktoren der Bildungsausgaben entwickelt wurden, sollen diese nun einem empirischen Test unterzogen werden. Für Variablendefinitionen und verwendete Quellen verweise ich auf den Anhang. ${ }^{9}$ Die Untersuchung umfasst 21 OECD-Länder und

9 Im Online-Anhang findet sich auch eine kurze Beschreibung (Mittelwerte, Standardabwei- 
erstreckt sich, wenn nicht anders angegeben, über den Zeitraum von 1980 bis 2001, für die privaten Ausgaben von 1993 bis 2001.

Die verwendete statistische Methode ist die gepoolte Zeitserienanalyse, in der es um die Kombination aus Quer- und Längsschnittbetrachtung geht. Das verwendete Standard-Modell orientiert sich an den Vorschlägen von Beck und Katz $(1995,1996)$, d.h. zur Berechnung der Standardfehler (und der damit einhergehenden t- bzw. z-Statistiken) wurden "panel-corrected standard errors" (PCSE) ${ }^{10}$ verwendet und zur Beseitigung der Autokorrelation wurde das Ausgabenniveau der Vorperiode („lagged dependent variable") in das Modell eingeschlossen. Zur Überprüfung der Robustheit der Ergebnisse wurden auch alternative Modellspezifikationen getestet. ${ }^{11}$

\section{2 Öffentliche Bildungsausgaben}

Tabelle 1 präsentiert die Ergebnisse der quantitativen Analyse der öffentlichen Bildungsausgabenquoten. Die ersten sechs Variablen bilden dabei die Basisspezifikation: Hierbei handelt es sich um diejenigen Bestimmungsfaktoren, die sich beim Durchrechnen verschiedenster Modellspezifikationen als sehr robust erwiesen haben. Dabei handelt es sich im Einzelnen um folgende Variablen:

Das Ausgabenniveau der Vorperiode (Höhe der Bildungsausgabenquote im Vorjahr) ist wie erwartet stark positiv mit den aktuellen Ausgaben assoziiert. Die Größe des Effektes ist beträchtlich: Jeder Prozentpunkt der Vorperiodenausgaben „erklärt“ bereits gut 0,86 Prozentpunkte des aktuellen Niveaus. Die Dominanz der lagged dependent variable über die anderen unabhängigen Variablen ist aus der statistischen Methodenlehre wohlbekannt (Kittel/Winner 2002).

Das BIP pro Kopf $f$ ist ebenfalls positiv mit den öffentlichen Bildungsausgaben assoziiert. Im Unterschied zu Castles' (1989) Befunden kann also die Gültigkeit des Wagnerschen Gesetzes auch im Falle der Bildungsausgaben bestätigt werden. Ein Anstieg des BIP pro Kopf um 1.000 Dollar ist demnach assoziiert mit einem Anstieg der Bildungsausgabenquote um knapp 0,02 Prozentpunkte. Wenn man zum Beispiel Deutschland

chung, Verlauf über die Zeit) der betrachteten abhängigen und der wichtigsten unabhängigen Variablen.

10 Im Speziellen kam der Befehl „xtpcse“ des Stata-Programms zum Einsatz.

11 So wurde anstelle des PCSE-Modells mit "lagged dependent variable“ (LDV) ein GLS-Modell mit AR(1)-Fehlerkorrekturprozess eingesetzt. Die Vorzeichen blieben dabei im Wesentlichen dieselben, auch wenn die t-Werte insgesamt geringer ausfielen. Insbesondere habe ich mich gegen die Verwendung eines "fixed effects"-Modells entschieden. Dabei geht es nicht nur darum, dass dadurch die Inklusion institutioneller Variablen, die sich über die Zeit hinweg nicht verändern und daher leicht von den "fixed effects" dominiert werden können, erschwert wird. Mit der "fixed effects"-Spezifizierung geht auch eine signifikante Veränderung der Fragestellung einher, die sich massiv auf die Interpretation der berechneten Regressionskoeffizienten niederschlägt. Die „fixed effects" bereinigen das Modell von aller Variation, die über die Zeit hinweg nicht veränderlich ist und systematisch mit den einzelnen Ländern zusammenhängt. Dadurch verschiebt sich die Betrachtungsperspektive noch weiter weg von der Querschnitt- oder der kombinierten Quer- und Längsschnitt- hin zur einfachen Längsschnittbetrachtung („within effects model“). Mir geht es aber um die Schätzung eines Effektes, der sowohl auf der Längs- als auch auf der Querschnittdimension beruht („pooled estimator“). Daher kommen in dem hier verwendeten Standardmodell keine „fixed effets" zum Einsatz. Vgl. aber die Tabellen mit verschiedenen Modellspezifikationen im Online-Anhang. 
Tabelle 1: Gepoolte Zeitserienanalyse der öffentlichen Bildungsausgaben in 21 OECDLändern, 1980-2001

\begin{tabular}{|c|c|c|c|c|c|}
\hline & \multicolumn{5}{|c|}{ Öffentliche Bildungsausgaben in \% des BIP } \\
\hline & Modell 1 & Modell 2 & Modell 3 & Modell 4 & Modell 5 \\
\hline $\begin{array}{l}\text { Bildungsausgaben des } \\
\text { Vorjahres (LDV) }\end{array}$ & $\begin{array}{c}0.858 \\
(56.23)^{* *}\end{array}$ & $\begin{array}{c}0.860 \\
(55.50)^{* *}\end{array}$ & $\begin{array}{c}0.864 \\
(50.36)^{* *}\end{array}$ & $\begin{array}{c}0.865 \\
(26.41)^{* *}\end{array}$ & $\begin{array}{c}0.857 \\
(32.40)^{* *}\end{array}$ \\
\hline $\begin{array}{l}\text { BIP pro Kopf } \\
\text { (in } 1000 \text { Dollar) }\end{array}$ & $\begin{array}{l}0.015 \\
(3.29)^{* *}\end{array}$ & $\begin{array}{c}0.014 \\
(3.24)^{* *}\end{array}$ & $\begin{array}{c}0.017 \\
(4.10)^{* *}\end{array}$ & $\begin{array}{c}0.018 \\
(3.36)^{* *}\end{array}$ & \\
\hline Wachstums des realen BIP & $\begin{array}{l}-0.047 \\
(10.16)^{* *}\end{array}$ & $\begin{array}{l}-0.047 \\
(10.23)^{* *}\end{array}$ & $\begin{array}{l}-0.041 \\
(6.21)^{* *}\end{array}$ & $\begin{array}{l}-0.038 \\
(4.32)^{* *}\end{array}$ & $\begin{array}{l}-0.039 \\
(4.85)^{* *}\end{array}$ \\
\hline $\begin{array}{l}\text { Anteil der 5- bis 29-Jähri- } \\
\text { gen an der Bevölkerung }\end{array}$ & $\begin{array}{l}0.020 \\
(5.25)^{* *}\end{array}$ & $\begin{array}{l}0.019 \\
(5.31)^{* *}\end{array}$ & $\begin{array}{l}0.025 \\
(4.96)^{* *}\end{array}$ & $\begin{array}{c}0.032 \\
(4.51)^{* *}\end{array}$ & $\begin{array}{l}0.029 \\
(4.30)^{* *}\end{array}$ \\
\hline Veto-Index & $\begin{array}{l}-0.013 \\
(2.15)^{*}\end{array}$ & $\begin{array}{c}-0.013 \\
(2.23)^{*}\end{array}$ & $\begin{array}{l}-0.019 \\
(3.38)^{* *}\end{array}$ & $\begin{array}{l}-0.018 \\
(2.51)^{*}\end{array}$ & \\
\hline Öff. Sozialausgabenquote & $\begin{array}{c}0.023 \\
(3.28)^{* *}\end{array}$ & $\begin{array}{c}0.021 \\
(3.76)^{* *}\end{array}$ & $\begin{array}{l}0.017 \\
(6.08)^{* *}\end{array}$ & $\begin{array}{l}0.021 \\
(4.23)^{* *}\end{array}$ & $\begin{array}{l}0.015 \\
(4.30)^{* *}\end{array}$ \\
\hline Öff. Ausgaben für Rente & $\begin{array}{c}-0.018 \\
(1.57)\end{array}$ & & & & \\
\hline $\begin{array}{l}\text { Öff. Ausgaben für } \\
\text { Arbeitslosigkeit }\end{array}$ & $\begin{array}{r}-0.027 \\
(1.35)\end{array}$ & & & & \\
\hline $\begin{array}{l}\text { Öff. Ausgaben für Aktive } \\
\text { Arbeitsmarktspolitik }\end{array}$ & $\begin{array}{c}-0.002 \\
(0.08)\end{array}$ & & & & \\
\hline Öff. Ausgaben für Familie & $\begin{array}{r}0.000 \\
(0.02)\end{array}$ & & & & \\
\hline $\begin{array}{l}\text { Summe aus Ausgaben für } \\
\text { Rente und Arbeitslosigkeit }\end{array}$ & & $\begin{array}{c}-0.016 \\
(1.63)\end{array}$ & & & \\
\hline $\begin{array}{l}\text { Summe aus Ausgaben für } \\
\text { Familie und AAMP }\end{array}$ & & $\begin{array}{l}0.002 \\
(0.21)\end{array}$ & & & \\
\hline $\begin{array}{l}\text { Stand der öff. Verschuldung } \\
\text { in Prozent des BIP }\end{array}$ & & & $\begin{array}{c}-0.001 \\
(1.64)\end{array}$ & & \\
\hline $\begin{array}{l}\text { Stand der Bildungsausgaben } \\
\text { in den 1970ern }\end{array}$ & & & & $\begin{array}{r}-0.027 \\
(1.83)\end{array}$ & \\
\hline $\begin{array}{l}\text { Grad der fiskalischen Dezentra- } \\
\text { lisierung der Steuereinnahmen }\end{array}$ & & & & $\begin{array}{c}0.002 \\
(2.00)^{*}\end{array}$ & \\
\hline Frauenerwerbsquote & & & & & $\begin{array}{c}0.008 \\
(3.71)^{* *}\end{array}$ \\
\hline Konstante & $\begin{array}{c}-0.403 \\
(2.05)^{*}\end{array}$ & $\begin{array}{c}-0.332 \\
(1.79)\end{array}$ & $\begin{array}{l}-0.615 \\
(2.71)^{* *}\end{array}$ & $\begin{array}{l}-0.907 \\
(3.36)^{* *}\end{array}$ & $\begin{array}{l}-1.041 \\
(3.54)^{* *}\end{array}$ \\
\hline $\mathrm{N}$ & 363 & 363 & 396 & 428 & 434 \\
\hline Zahl der Länder & 21 & 21 & 20 & 21 & 21 \\
\hline
\end{tabular}

Quelle: Bildungsausgaben: OECD (verschiedene Jahrgänge): Education at a Glance, OECD (1992). BIP pro Kopf: Maddison (2003). Wirtschaftswachstum (real): OECD Economic Outlook Database. Anteil der 5-29-Jährigen an der Bevölkerung. OECD Education at a Glance, eigene Berechnungen auf der Grundlage von UNESCO (verschiedene Jahrgänge): Statistical Yearbook. Veto-Index. 


\section{Fortsetzung Tabelle 1}

Schmidt (2000: 352-353). Sozialausgaben: OECD Social Expenditure Database. Stand der öffentlichen Verschuldung. OECD Economic Outlook Database. Grad der Dezentralisierung der Steuereinnahmen: Stegarescu (2004).

Angaben: Panel-corrected z-Statistiken in Klammern; * signifikant auf Fünf-Prozent-Niveau; ${ }^{* *}$ signifikant auf Ein-Prozent-Niveau; Definitionen der Ausgabenkategorien „Rente“, „Familie“, „Arbeitslosigkeit" und „aktive Arbeitsmarktpolitik" richten sich nach den Definitionen der OECD Social Expenditure Database. Die Zahl der Fälle reduziert sich in den ersten beiden Modellen aufgrund von fehlenden Daten zu den Ausgaben für aktive Arbeitsmarktpolitik. Die Robustheit des Basismodells hinsichtlich der statistischen Signifikanz und der Größe der Schätzer bleibt aber auch bei leicht erhöhter Fallzahl gegeben.

mit den USA vergleicht, zwischen denen beim um Unterschiede in der Kaufkraft bereinigten Indikator BIP pro Kopf ungefähr 10.000 Dollar liegen, so kann dieser Unterschied 0,2 Prozentpunkte des Unterschiedes in der Bildungsausgabenquote erklären.

Zwischen dem Wirtschaftswachstum und den öffentlichen Bildungsausgaben findet sich eine negative Assoziation. Dabei könnte es sich zum einen um ein statistisches Artefakt handeln, denn dadurch, dass das BIP sowohl im Nenner der Bildungsausgabenquote als auch als Bezugsgröße der Variablen Wirtschaftswachstum auftaucht, resultiert aus einem Ansteigen des BIP (Wachstum) ein Absinken der Quote, ohne dass sich die absolute Höhe der Ausgaben verändert. Zum anderen ist die negative Assoziation aber auch ein Hinweis darauf, dass die öffentlichen Bildungsausgaben im Gegensatz zu Sozialausgaben von den Schwankungen des ökonomischen Konjunkturzyklus relativ unbeeinträchtigt bleiben.

Der Anteil der 5- bis 29-Jährigen an der Bevölkerung ist positiv mit den öffentlichen Bildungsausgaben assoziiert. Die pessimistische These der „Generational Politics“ erhält hier also einen ersten Dämpfer: Zumindest was die Ausgabenniveaus angeht, geben Staaten mit einem höheren Bevölkerungsanteil junger Leute mehr für Bildung aus. ${ }^{12}$

Der Grad der Ausprägung der konstitutionellen Vetostruktur hat sich als negativer Bestimmungsfaktor der öffentlichen Bildungsausgaben herausgestellt. Weiterhin lässt sich eine positive Beziehung zwischen den öffentlichen Bildungs- und den öffentlichen Sozialausgaben nachweisen. Diese beiden Befunde deuten darauf hin, dass die Institutionalisierung des öffentlichen Bildungsstaates in weiten Strecken Hand in Hand ging mit dem Auf- und Ausbau des Sozialstaates. Der positive Effekt, den eine Zentralisierung der politischen Steuerungsmacht bei der Zentralregierung auf die Expansion der Sozialausgaben gehabt hat, lässt sich auch für die öffentlichen Bildungsausgaben nachweisen.

Abgesehen von diesen robusten Erklärungsfaktoren des Basismodells sind im Weiteren andere unabhängige Variablen auf ihre Relevanz getestet worden. Dabei konnte die Existenz einer Programm- und Finanzierungskonkurrenz zwischen der Bildungspolitik und bestimmten Ausgabenfeldern der Sozialpolitik nachgewiesen werden. Die These, dass der Bildungs- und der Sozialstaat gemeinsam expandierten, trifft daher nur für be-

12 Nach den Ergebnissen der Tabelle 1 ist ein Unterschied im Anteil des Bevölkerungsanteils der Jungen von beispielsweise zehn Prozent verbunden mit einem Unterschied in der Bildungsausgabenquote von gut 0,2 Prozentpunkten. 
stimmte Wohlfahrtsstaaten zu. Insbesondere zeigt sich eine negative Beziehung zwischen den Ausgaben für Rente sowie der Summe aus Ausgaben für Rente und Arbeitslosigkeit einerseits und den öffentlichen Bildungsausgaben andererseits. Hohe konsumtive Transferzahlungen sind charakteristisch für die kontinentaleuropäischen Wohlfahrtsstaaten. Die Ergebnisse der Tabelle 1 belegen, dass dadurch die Ausgaben für sozialinvestive Politiken wie die Bildung zu kurz kommen. Die positive Assoziation zwischen den öffentlichen Bildungs- und Sozialausgaben sowie die Nicht-Signifikanz der Ausgaben für Familie und Aktive Arbeitsmarktpolitik deuten darauf hin, dass in universalistischen Wohlfahrtsstaaten, die durch insgesamt hohe Sozialausgaben und besondere Schwerpunktsetzung auf investive, aktivierende Politiken gekennzeichnet sind, der Bildung ebenfalls beträchtliche öffentliche Ressourcen zugeschrieben werden. Zwischen den öffentlichen Bildungsausgaben und den Indikatoren der Staatsverschuldung ${ }^{13}$ besteht eine negative Assoziation (signifikant auf dem Zehn-Prozent-Niveau). Dies belegt die oben diskutierte These, dass in Zeiten der knappen öffentlichen Mittel und der hohen Staatsverschuldung vor allem die Bildungspolitik zu leiden hat. ${ }^{14}$

Das negative Vorzeichen des Regressionskoeffizienten der Variablen „Stand der Bildungsausgaben in den 1970er Jahren" deutet auf die Präsenz eines Catch-up-Effektes hin (Zehn-Prozent-Signifikanzniveau). Das heißt, eine negative Beziehung zwischen den Ausgabenniveaus der 1970er und 1980er bzw. 1990er Jahre bedeutet, dass die Länder, die in den 1970er Jahren wenig ausgegeben haben, heute viel ausgeben und bzw. oder umgekehrt. Die fiskalische Dezentralisierung hängt positiv mit der durchschnittlichen Höhe der Bildungsausgaben zusammen. Es findet sich also eine gewisse Evidenz für die Existenz eines lokalen Überbietungswettbewerbs in der Bildungspolitik, zumal der Dummy-Indikator für konstitutionell föderalistische Länder sich als nicht erklärungskräftig herausgestellt hat. Schließlich ist auch die Frauenerwerbsquote positiv mit den öffentlichen Bildungsausgaben assoziiert. ${ }^{15}$ Wie oben angedeutet, ist hier die Richtung des kausalen Zusammenhangs aber im Wesentlichen unklar.

In Tabelle 2 kann zumindest für die Subperiode der 1980er Jahre die Präsenz von parteipolitischen und Machtressourceneffekten nachgewiesen werden. ${ }^{16}$ Abgesehen von den bereits beschriebenen Variablen des Basismodells zeigt sich, dass die Regierungsbeteiligung linker (bürgerlicher) Parteien mit einem Ansteigen (Absinken) der öffentlichen Bildungsausgaben verbunden ist. Ein Wechsel von einer Regierung ohne Sozialdemokraten zu einer Regierung, die nur aus Sozialdemokraten besteht, geht demnach einher mit einem Ansteigen der Bildungsausgabenquote um 0,1 Prozentpunkte, also

13 In Tabelle 1: der Stand der Gesamtverschuldung in Prozent des BIP. Auch die Zinslastquote (Höhe der Zinszahlungen für Staatsschulden in Prozent des BIP) hat sich als ähnlich erklärungskräftig erwiesen.

14 Zwischen dem Stand der Verschuldung und den öffentlichen Sozialausgaben besteht eine positive Korrelation von ungefähr 0,2. Dies deutet darauf hin, dass die Sozialausgaben nicht im gleichen Maße von den Kürzungen betroffen sind wie die Bildungsausgaben, sondern im Gegenteil sogar zum Teil für den Anstieg der Staatsverschuldung verantwortlich gemacht werden können.

15 Aufgrund von Multikollinearitätsproblemen musste in diesem Modell das BIP pro Kopf und der Veto-Index ausgeschlossen werden.

16 Der Einfluss von Parteieffekten wurde auch für andere Zeitperioden untersucht, ist aber lediglich für die Subperiode der 1980er erklärungskräftig. 
Tabelle 2: Machtressourcen und Parteieneffekte in 19 OECD-Ländern, 1980-1989

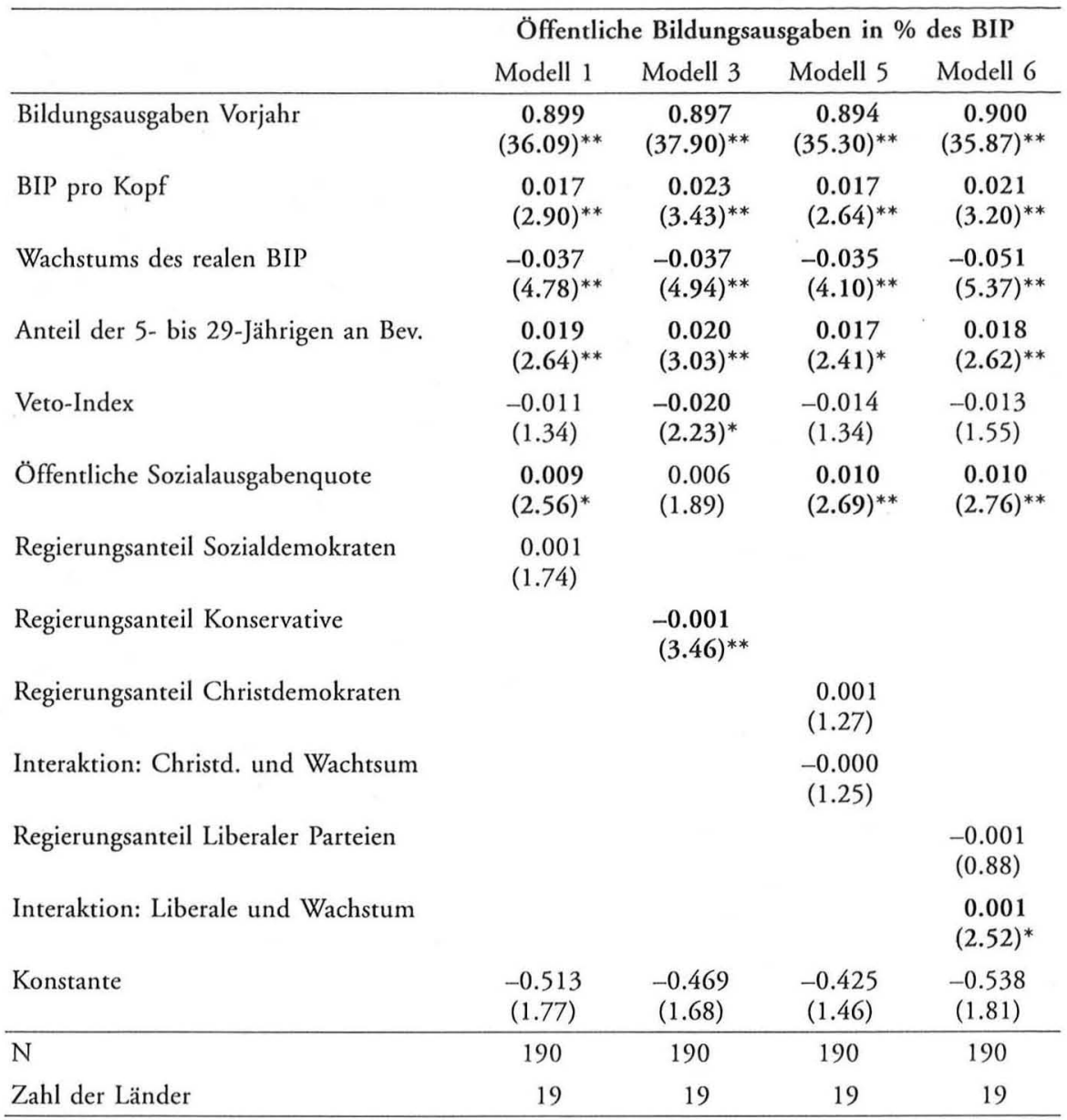

Quelle: Bildungsausgaben: OECD (verschiedene Jahrgänge): Education at a Glance, OECD (1992). BIP pro Kopf: Maddison (2003). Wirtschaftswachstum (real): OECD Economic Outlook Database. Anteil der 5-29-Jährigen an der Bevölkerung: OECD: Education at a Glance, eigene Berechnungen auf der Grundlage von UNESCO (verschiedene Jahrgänge): Statistical Yearbook. Veto-Index: Schmidt (2000: 352-353). Sozialausgaben: OECD Social Expenditure Database. Daten zur parteipolitischen Zusammensetzung der OECD-Regierungen: Schmidt (2003b).

Angaben: Panel-corrected z-Statistiken in Klammern, * signifikant auf Fünf-Prozent-Niveau; ${ }^{* *}$ signifikant auf Ein-Prozent-Niveau; Die Zahl der betrachteten Länder reduziert sich von 21 auf 19, weil für Dänemark und Spanien in den 1980er Jahren keine OECD-Daten für die öffentliche Bildungsausgabenquote verfügbar sind. 
etwa die Hälfte des Effektes, den eine Zunahme des BIP pro Kopf um 10.000 Dollar verursacht. Für die konservative Parteifamilie ist ein ebenso großer negativer Effekt nachweisbar, der zudem in höherem Maße statistisch signifikant ist.

Bei den Mitteparteien ist die Lage nicht so eindeutig. Für die liberale Parteifamilie bestätigt sich die Prosperitätsthese: Lediglich in Zeiten des wirtschaftlichen Wachstums lässt sich ein positiver Zusammenhang zwischen der Regierungsbeteiligung Liberaler und den öffentlichen Bildungsausgaben nachweisen. Dies könnte auch daran liegen, dass es den meist in Minderheitenposition an der Regierung beteiligten Liberalen nur in wirtschaftlichen Boomzeiten gelingt, ihre Koalitionspartner von der Notwendigkeit der Investitionen in Bildung zu überzeugen. Bei den Christdemokraten führt die Inklusion der Interaktionsvariablen aus Wirtschaftswachstum und Regierungsbeteiligung dazu, dass der Effekt der einfachen Regierungsbeteiligungsvariablen, der vorher nicht signifikant war, positiv wird. Dies könnte ein Beleg sein für die allgemeine Ausgabenfreudigkeit der Christdemokraten. Die negative Assoziation zu der Interaktionsvariablen deutet allerdings an, dass in Zeiten des wirtschaftlichen Wachstums der Ausbau des öffentlichen Bildungsstaates nicht unbedingt zu den Prioritäten der Christdemokraten gehört.

\subsection{Private Bildungsausgaben}

In Tabelle 3 sind die Ergebnisse der Analyse der privaten Bildungsausgabenquoten dargestellt. Aufgrund der Datenverfügbarkeit reduziert sich der Beobachtungszeitraum auf die Periode zwischen 1993 und 2001. Belgien, Griechenland, Neuseeland, Norwegen, die Schweiz und in einigen Analysen auch Frankreich mussten aufgrund von fehlenden Daten ausgeschlossen werden. Dennoch lassen sich einige interessante Ergebnisse präsentieren.

Es zeigt sich, dass die privaten Ausgaben durch drei demographische Determinanten geprägt sind: Die Frauenerwerbsquote, die Bildungspartizipation im tertiären Sektor und der Anteil der Beschäftigten im industriellen Sektor an der Gesamtbeschäftigung sind alle stark positiv mit den privaten Ausgaben assoziiert. Ein Vergleich der absoluten Größe der Effekte zeigt, dass ein Anstieg um beispielsweise zehn Prozentpunkte in der Bildungspartizipation im tertiären Sektor mit einem Ausgabenanstieg von 0,07 Prozentpunkten des BIP-Anteils einhergeht (ca. zehn Prozent des OECD-Durchschnitts der privaten Bildungsausgaben: 0,9 Prozent), während ein Ansteigen der Frauenerwerbsquote und des Anteils der im industriellen Sektor Beschäftigten um denselben Betrag mit einem Anstieg der privaten Ausgaben von ca. 0,14 Prozentpunkten einhergeht. Damit sind die zentralen demographischen Triebkräfte der privaten Bildungsausgaben identifiziert: Ein ausgebauter Hochschulsektor, ein die Frauenerwerbsbeteiligung fördernder Arbeitsmarkt sowie ein hoher Anteil der im industriellen Sektor Beschäftigten fördern die privaten Bildungsausgaben. Eine genauere Aufschlüsselung nach Bildungssektoren könnte hier mehr Aufschluss geben: Zeitreihen zu privaten sektoralen Bildungsausgaben sind leider kaum verfügbar, aber es fällt auf, dass im internationalen Vergleich der privaten, post-sekundären Bildungsausgaben vor allem die Länder mit dualem System der Berufsausbildung (Deutschland, Schweiz) und bzw. oder einem 
Tabelle 3: Determinanten der privaten Bildungsausgabenquote in 16 OECD-Ländern, 1993-2001

\begin{tabular}{|c|c|c|c|}
\hline & \multicolumn{3}{|c|}{ Private Bildungsausgaben in \% des BIP } \\
\hline & Modell 1 & Modell 2 & Modell 3 \\
\hline Bildungsausgaben Vorperiode & $\begin{array}{c}0.649 \\
(10.72)^{* *}\end{array}$ & $\begin{array}{c}0.693 \\
(12.00)^{* *}\end{array}$ & $\begin{array}{l}0.619 \\
(8.91)^{* *}\end{array}$ \\
\hline Öffentliche Sozialausgaben & $\begin{array}{l}-0.026 \\
(5.16)^{* *}\end{array}$ & $\begin{array}{l}-0.028 \\
(5.67)^{* *}\end{array}$ & $\begin{array}{l}-0.018 \\
(5.16)^{* *}\end{array}$ \\
\hline $\begin{array}{l}\text { Interaktion Veto } \times \text { Anteil der } 5 \text { - bis } \\
\text { 29-Jährigen }\end{array}$ & $\begin{array}{l}0.011 \\
(4.98)^{* *}\end{array}$ & $\begin{array}{l}0.011 \\
(4.55)^{* *}\end{array}$ & $\begin{array}{l}0.016 \\
(3.67)^{* *}\end{array}$ \\
\hline Bildungspartizipation im Tertiärsektor & $\begin{array}{l}0.007 \\
(4.39)^{* *}\end{array}$ & & $\begin{array}{l}0.006 \\
(3.71)^{* *}\end{array}$ \\
\hline $\begin{array}{l}\text { Beschäftigtenanteil im Industriesektor an } \\
\text { Gesamtbeschäftigten }\end{array}$ & $\begin{array}{l}0.012 \\
(3.60)^{* *}\end{array}$ & & $\begin{array}{l}0.015 \\
(3.84)^{* *}\end{array}$ \\
\hline Frauenerwerbsquote & $\begin{array}{l}0.008 \\
(4.58)^{* *}\end{array}$ & $\begin{array}{l}0.010 \\
(4.51)^{* *}\end{array}$ & $\begin{array}{l}0.014 \\
(3.46)^{* *}\end{array}$ \\
\hline Bevölkerungsanteil 5- bis 29-Jährige & & $\begin{array}{l}-0.012 \\
(2.56)^{*}\end{array}$ & \\
\hline Integrationsindex (Siaroff) & & & $\begin{array}{c}0.207 \\
(2.26)^{*}\end{array}$ \\
\hline Siaroff-Index quadriert & & & $\begin{array}{c}-0.044 \\
(2.29)^{*}\end{array}$ \\
\hline Konstante & $\begin{array}{c}-0.644 \\
(3.92)^{* *}\end{array}$ & $\begin{array}{c}0.399 \\
(1.78) \\
\end{array}$ & $\begin{array}{c}-1.412 \\
(3.36)^{* *}\end{array}$ \\
\hline $\mathrm{N}$ & 112 & 138 & 112 \\
\hline Zahl der Länder & 15 & 16 & 15 \\
\hline
\end{tabular}

Quelle: Bildungsausgaben: OECD (verschiedene Jahrgänge): Education at a Glance, sowie OECD (1992). BIP pro Kopf: Maddison (2003). Wirtschaftswachstum (real): OECD (verschiedene Jahrgänge): Economic Outlook Database. Anteil der 5-29-Jährigen an der Bevölkerung. OECD (verschiedene Jahrgänge): Education at a Glance, eigene Berechnungen auf der Grundlage von UNESCO (verschiedene Jahrgänge): Statistical Yearbook. Veto-Index: Schmidt (2000: 352-353). Sozialausgaben: OECD Social Expenditure Database. Integrationsindex: Siaroff (1999). Frauenerwerbsbeteiligung und Beschäftigtenanteil im Industriesektor: OECD (verschiedene Jahrgänge): Labour Force Statistics.

Angaben: Panel-corrected z-Statistiken in Klammern; * signifikant auf Fünf-Prozent-Niveau; ${ }^{* *}$ signifikant auf Ein-Prozent-Niveau; Belgien, Griechenland, Neuseeland, Norwegen, Schweiz mussten auf Grund mangelnder Daten in der abhängigen Variable ausgeschlossen werden, z.T. auch Frankreich aufgrund mangelnder Daten in einigen wichtigen unabhängigen Variablen (hauptsächlich Anteil der im Industriesektor Beschäftigten an Gesamtbeschäftigung).

ausgebauten privaten Hochschulsektor (USA, Kanada) bzw. privater Hochschulfinanzierung (Japan) hervorstechen.

Neben den demographischen Variablen haben sich aber auch einige politisch-institutionelle Variablen als erklärungskräftig erwiesen. Zwischen der öffentlichen Sozialleistungsquote und den privaten Bildungsausgaben lässt sich eine negative Assoziation 
nachweisen. Das deutet darauf hin, dass fehlende öffentliche Sozialleistungen vor allem durch private Investitionen in den Bildungssektor zum Teil kompensiert werden. Diese Tatsache muss vor dem Hintergrund unterschiedlicher sozial- und bildungspolitischer Traditionen gesehen werden. Es bleibt näher zu erforschen, inwiefern diese unterschiedlichen Schwerpunktsetzungen in den wohlfahrtsstaatlichen Institutionen und dem System der Staat-Markt-Arbeitsteilung mit unterschiedlichen Gerechtigkeitsvorstellungen (Chancengleichheit vs. Gleichheit der Outcomes) einhergehen. Der positive Koeffizient der Interaktionsvariablen „Veto-Index“ $\times$ „Bevölkerungsanteil der 5- bis 29Jährigen“ (vgl. Tabelle 3) jedenfalls deutet darauf hin, dass eine ausgebaute Vetostruktur, die für den Ausbau des öffentlichen Sozialstaates eine Bremse war, bei Vorhandensein entsprechender demographischer Nachfrage mit höheren privaten Ausgabenniveaus einhergeht. Eine Vielzahl von konstitutionellen und semi-formellen Vetospielern mag die Ausgabenintensität des öffentlichen Wohlfahrtsstaates beschränkt haben, sie hat aber auch die Etablierung von privaten Alternativen gefördert, wenn die entsprechende Nachfrage nach Bildungsdienstleistungen nicht effektiv durch öffentliche Lösungen befriedigt werden konnte.

Zwischen den privaten Bildungsausgaben und dem Korporatismus-Indikator (Siaroff 1999) besteht eine nicht-lineare, umgekehrt U-förmige Assoziation. Die Nonlinearität ist vor allem dadurch begründet, dass Spanien und Portugal, die über geringe Privatausgaben verfügen, im Siaroff-Index geringere Werte zugewiesen bekommen als klassisch liberale Staaten wie die USA oder Kanada. Über den gesamten Verlauf der Variablen betrachtet besteht daher tendenziell eine eher negative Beziehung zwischen Korporatismus und privaten Ausgaben. Dies ist insofern interessant, als die Sozialpartner in hoch korporatistischen Systemen offenbar die Bereitstellung von Bildungsdienstleistungen über die öffentliche Sphäre der zwischen privater und öffentlicher Hand geteilten Verantwortung vorziehen.

\subsection{Sektorale Bildungsausgaben}

Abschließend seien noch die Ergebnisse der Analysen der sektoralen öffentlichen Bildungsausgaben präsentiert (Tabelle 4). Generell hat sich herausgestellt, dass die Ausgaben für den Primär- und Sekundärsektor ${ }^{17}$ im internationalen Vergleich nicht so stark variieren wie die Ausgaben für den post-sekundären und Tertiärbereich (Variationskoeffizienten von 0.17 bzw. 0.35). Die Bereitstellung einer universalen Grund- und Sekundarschulbildung gehört heute zum Kernbestand der modernen Industrienationen. Die Förderung des Ausbaus des Hochschulsektors ist jedoch im Gegensatz dazu stärker durch politische Faktoren bestimmt.

Tabelle 4 zeigt, dass die Identifizierung von Bestimmungsfaktoren der Primär- und Sekundärbildungsausgaben keine leichte Aufgabe ist. Abgesehen von der Ausgabenhöhe der Vorperiode haben sich lediglich die jeweilige sektorale Bildungspartizipation sowie das Zahlenverhältnis zwischen Schülern und Lehrern als mittelmäßig erklärungskräftig erwiesen. Je höher der Anteil der Bildungsteilnehmer an der jeweils relevanten Alters-

17 Aus Gründen der Datenverfügbarkeit sind nur die Betrachtungen der summierten Ausgaben für den Primär- und Sekundärsektor möglich. 
Tabelle 4: Determinanten der sektoralen öffentlichen Bildungsausgaben, 1991-2001

\begin{tabular}{|c|c|c|c|c|c|}
\hline & \multicolumn{2}{|c|}{$\begin{array}{l}\text { Öffentliche Bildungs- } \\
\text { ausgaben im Primär- } \\
\text { und Sekundärsektor } \\
\text { in \% des BIP }\end{array}$} & \multicolumn{3}{|c|}{$\begin{array}{l}\text { Öffentliche Bildungsausgaben im } \\
\text { Tertiärsektor in \% des BIP }\end{array}$} \\
\hline & Modell 1 & Modell 2 & Modell 3 & Modell 4 & Modell 5 \\
\hline $\begin{array}{l}\text { Primär-/Sekundärausgaben } \\
\text { des Vorjahres }\end{array}$ & $\begin{array}{c}0.867 \\
(13.87)^{* *}\end{array}$ & $\begin{array}{c}0.875 \\
(\mathbf{1 8 . 8 4})^{* *}\end{array}$ & & & \\
\hline $\begin{array}{l}\text { Zahlenverhältnis Schüler- } \\
\text { Lehrer im Primärsektor }\end{array}$ & $\begin{array}{r}-0.007 \\
(1.71)\end{array}$ & & & & \\
\hline $\begin{array}{l}\text { Bildungspartizipation im } \\
\text { Primärsektor }\end{array}$ & $\begin{array}{l}0.003 \\
(1.95)\end{array}$ & & & & \\
\hline $\begin{array}{l}\text { Zahlenverhältnis Schüler- } \\
\text { Lehrer im Sekundärsektor }\end{array}$ & & $\begin{array}{c}-0.004 \\
(1.03)\end{array}$ & & & \\
\hline $\begin{array}{l}\text { Bildungspartizipation im } \\
\text { Sekundärsektor }\end{array}$ & & $\begin{array}{c}0.001 \\
(1.39)\end{array}$ & & & \\
\hline $\begin{array}{l}\text { Tertiärausgaben des } \\
\text { Vorjahres }\end{array}$ & & & $\begin{array}{l}0.494 \\
(3.80)^{* *}\end{array}$ & $\begin{array}{l}0.481 \\
(3.65)^{* *}\end{array}$ & $\begin{array}{l}0.639 \\
(8.54)^{* *}\end{array}$ \\
\hline $\begin{array}{l}\text { Offenheit der } \\
\text { Volkswirtschaft }\end{array}$ & & & $\begin{array}{c}0.003 \\
(3.60)^{* *}\end{array}$ & $\begin{array}{c}0.003 \\
(3.76)^{* *}\end{array}$ & $\begin{array}{l}0.002 \\
(3.68)^{* *}\end{array}$ \\
\hline $\begin{array}{l}\text { Anteil der 5-bis 29-Jäh- } \\
\text { rigen an Bevölkerung }\end{array}$ & & & $\begin{array}{r}-0.007 \\
(1.88)\end{array}$ & $\begin{array}{c}-0.006 \\
(1.30)\end{array}$ & $\begin{array}{l}-0.021 \\
(4.48)^{* *}\end{array}$ \\
\hline $\begin{array}{l}\text { Bildungspartizipation im } \\
\text { Tertiärsektor }\end{array}$ & & & $\begin{array}{l}0.006 \\
(3.15)^{* *}\end{array}$ & $\begin{array}{l}0.006 \\
(2.95)^{* *}\end{array}$ & $\begin{array}{l}0.004 \\
(3.81)^{* *}\end{array}$ \\
\hline $\begin{array}{l}\text { Beschäftigtenanteil im Indu } \\
\text { sektor an Gesamtbeschäftigt }\end{array}$ & & & & & $\begin{array}{l}-0.007 \\
(2.04)^{*}\end{array}$ \\
\hline Frauenerwerbsbeteiligung & & & $\begin{array}{l}0.010 \\
(4.31)^{* *}\end{array}$ & $\begin{array}{l}0.010 \\
(4.59)^{* *}\end{array}$ & \\
\hline $\begin{array}{l}\text { Kabinettsitzanteil } \\
\text { Konservative }\end{array}$ & & & & $\begin{array}{l}-0.001 \\
(3.69)^{* *}\end{array}$ & \\
\hline $\begin{array}{l}\text { Kabinettsitzanteil } \\
\text { Christdemokraten }\end{array}$ & & & & $\begin{array}{l}-0.001 \\
(2.71)^{* *}\end{array}$ & \\
\hline $\begin{array}{l}\text { Kabinettsitzanteil } \\
\text { Sozialdemokraten }\end{array}$ & & & $\begin{array}{c}0.001 \\
(3.16)^{* *}\end{array}$ & & \\
\hline Konstante & $\begin{array}{c}0.264 \\
(1.01) \\
\end{array}$ & $\begin{array}{c}0.352 \\
(1.78) \\
\end{array}$ & $\begin{array}{l}-0.382 \\
(1.99)^{*}\end{array}$ & $\begin{array}{r}-0.328 \\
(1.82) \\
\end{array}$ & $\begin{array}{l}0.948 \\
(3.62)^{* *}\end{array}$ \\
\hline $\mathrm{N}$ & 174 & 167 & 169 & 169 & 152 \\
\hline Zahl der Länder & 20 & 20 & 20 & 20 & 19 \\
\hline
\end{tabular}

Quelle: Bildungsausgaben: OECD (verschiedene Jahrgänge): Education at a Glance, OECD (1992). BIP pro Kopf: Maddison (2003). Wirtschaftswachstum (real): OECD Economic Outlook Database. Anteil der 5-29-Jährigen an der Bevölkerung: OECD (verschiedene Jahrgänge): Education at a Glance, eigene Berechnungen auf der Grundlage von UNESCO (verschiedene Jahrgänge): Statistical Yearbook. Veto-Index: Schmidt (2000: 352-353). Sozialausgaben: OECD Social Expenditure Database. Integrationsindex Siaroff (1999). Frauenerwerbsbeteiligung und Beschäftigtenanteil im Industriesektor: OECD (verschiedene Jahrgänge): Labour Force Statistics. Daten zur parteipolitischen 
Zusammensetzung der OECD-Regierungen: Schmidt (2003b). Offenheit der Volkswirtschaft: OECD (verschiedene Jahrgänge): Historical Statistics. Bildungspartizipation: UNESCO (verschiedene Jahrgänge): Statistical Yearbook.

Angaben: Spanien musste aufgrund mangelnder Daten in der abhängigen Variablen aus der Untersuchung ausgeschlossen werden.

gruppe (Bildungspartizipation), desto höher die Ausgaben. Und: Je mehr Schüler von einem Lehrer betreut werden müssen, desto niedriger die Ausgaben. Dieser Befund hat sich auch in einer Querschnittbetrachtung der durchschnittlichen Klassengröße und der sektoralen Ausgaben bestätigt. Staaten, die weniger ausgeben für Primär- und Sekundärschulbildung sind demnach durch größere Klassen gekennzeichnet.

Interessanter ist die Betrachtung der Determinanten der öffentlichen Ausgaben für Tertiärbildung. Erwartungsgemäß ist auch hier die sektorale Bildungspartizipation (grob gesagt der Anteil der Studenten an der Bevölkerungsgruppe der Jungen) ein wichtiger Antriebsfaktor der öffentlichen Ausgaben. Aber: Der Bevölkerungsanteil der 5- bis 29Jährigen ist negativ mit den öffentlichen Ausgaben für das Hochschulwesen assoziiert. Aus Abschnitt 3.2 wissen wir, dass diese Variable mit der Gesamthöhe der öffentlichen Ausgaben positiv zusammenhängt. Dieser teilweise verwirrende Befund lässt sich zum Teil durch die mittelstarke Korrelation $(-0.3)$ der tertiären Bildungspartizipation mit dem BIP pro Kopf erklären. Das heißt, in ärmeren Staaten ist der Bevölkerungsanteil der Jüngeren höher, die öffentlichen Ausgaben für den Tertiärsektor allerdings niedriger. Insofern erfasst diese Variable zum Teil auch die Auswirkungen der Bevölkerungsalterung in den reicheren, post-industriellen OECD-Demokratien. Der positive Effekt dieser Variablen, der sich bei der Analyse der gesamten öffentlichen Ausgabenquote gezeigt hat, ist daher im Wesentlichen auf die Dynamik der nicht-hochschulischen Bildungssektoren zurückzuführen.

Die internationale Hypothese, die von einem Zusammenhang zwischen der ökonomischen Globalisierung und den Staatsausgaben ausgeht, findet bei der Betrachtung der Tertiärausgaben eine Renaissance. Bei der Betrachtung der allgemeinen öffentlichen Bildungsausgaben war sie nicht erklärungskräftig. Hier jedoch zeigt sich, dass offene Volkswirtschaften auch über hohe öffentliche Tertiärausgaben verfügen. ${ }^{18}$

Des Weiteren findet sich ein robuster positiver Effekt auf die Ausgaben, der von der Regierungsbeteiligung sozialdemokratischer Parteien herrührt. Die Regierungsbeteili-

18 Die Offenheit der Volkswirtschaften ist dabei aber nicht assoziiert mit deren Offenheit für Studenten aus anderen Ländern $(r=0.13$, nicht signifikant, Indikator für relative Last durch ausländische Studierende aus OECD 2005: 267). Im Fall Deutschland ist beobachtet worden, dass es aufgrund der hohen Mobilität von Studierenden zwischen Hochschulstandorten in verschiedenen Bundesländern zu einer ineffizienten Bereitstellung von Bildungsleistungen (Unterversorgung) kommt (Büttner/Schwager 2004). Die internationale Mobilität der Studierenden hat zwar in den letzten Jahren zugenommen, aber noch nicht die Ausmaße der innerdeutschen Mobilität erreicht. Außerdem werden in einigen Ländern, die in besonderem Maße Zielland für internationale Studentenbewegungen sind oder einen besonders hohen Anteil ausländischer Studierender aufweisen (Australien, Österreich, Neuseeland, UK, USA), für Ausländer höhere Studiengebühren als für Inländer erhoben, sodass eine langfristige Erodierung der Steuerbasis vermieden werden kann (vgl. OECD 2005: 250ff.). 
gung Konservativer oder von Christdemokraten ist dagegen negativ mit den öffentlichen Hochschulausgaben assoziiert. Die Quintessenz dieser Befunde ist, dass das Instrument der staatlichen Investitionen in Humankapitalbildung im Hochschulsektor eine insbesondere von sozialdemokratischen Parteien in offenen Volkswirtschaften betriebe Konter-Strategie gegen die Auswirkungen der Globalisierung ist. Diese These ist auch schon von Boix $(1997,1998)$ sowie Garrett und Lange (1991) vertreten worden. Die klassische Kompensationsthese, in der der Wohlfahrtsstaat als Dämpfer potenzieller negativer sozialer Nebenwirkungen der Globalisierung angesehen wird, ist daher zu ergänzen um den Faktor Bildung: Die Unterstützung tertiärer Bildungsmöglichkeiten durch den Ausbau der öffentlichen Hochschulsysteme scheint eine besonders beliebte Strategie linker Regierungen im Kampf gegen die negativen Folgewirkungen der ökonomischen Globalisierung zu sein. Auch hier wird der eher sozialinvestive statt konsumtive Charakter der skandinavischen Wohlfahrtsstaaten deutlich.

Die Frauenerwerbsquote ist ebenfalls positiv mit den öffentlichen Ausgaben im Tertiärbereich assoziiert. Der tatsächliche kausale Zusammenhang zwischen Bildungsausgaben und Frauenerwerbsbeteiligung ist durch eine Regressionsanalyse aber nur begrenzt erfassbar, da diese primär Korrelationen und keine Kausalität widerspiegelt. Wie bereits oben angedeutet, ist zu vermuten, dass die Frauenerwerbsbeteiligung in stärkerem Maße als die anderen betrachteten unabhängigen Variablen in einer kausalen Wechselbeziehung mit den Bildungsausgaben steht. Einerseits ist der Ausbau des post-sekundären Bildungswesens, egal ob dies vordringlich durch die öffentliche oder die private Hand getan wird, eine Reaktion auf die gestiegene Bildungsnachfrage im Zuge der ansteigenden Frauenerwerbsbeteiligung. Andererseits eröffnet ein ausgebautes Bildungssystem neue Beschäftigungsmöglichkeiten, die auch für Frauen relevant sind. Besonders dieser Zusammenhang zwischen Frauenerwerbsbeteiligung und Bildungsausgaben sollte daher Gegenstand weiterer Forschungsbemühungen sein.

Erwartungsgemäß ist auch der Anteil der im industriellen Sektor Beschäftigten an der Gesamtbeschäftigung eine negative Determinante der öffentlichen Tertiärausgaben. Eine Ausrichtung der Volkswirtschaften auf den produzierenden, industriellen Sektor anstelle des Dienstleistungssektors geht mit einer geringeren Nachfrage nach qualifizierten Hochschulabgängern und daher auch niedrigeren öffentlichen Investitionen in das Universitätssystem einher.

\section{Fazit}

Dieser Aufsatz gab einen Überblick über die Determinanten der öffentlichen, privaten und sektoralen Bildungsausgaben im Vergleich der wirtschaftlich entwickelten OECDLänder. Insgesamt ist die Bedeutung von Programm- und Finanzierungskonkurrenzen vor allem zu sozialkonsumtiven Ausgabenfeldern wie der Renten- oder Arbeitslosenpolitik nachgewiesen worden. Außerdem konnte gezeigt werden, dass ökonomische und demographische Variablen eine hohe Erklärungskraft haben. Aber auch parteipolitische Variablen sind nicht zu vernachlässigen. Die Globalisierungshypothese hat sich vor allem zur Erklärung der öffentlichen Tertiärausgaben als hilfreich erwiesen. Allgemein gesprochen konnte ein gewisser Substitutionseffekt zwischen den öffentlichen und pri- 
vaten Bildungsausgaben nachgewiesen werden. Eine ausgebaute konstitutionelle Vetostruktur, die sich nicht nur auf die Sozial-, sondern auch auf die Entwicklung der Bildungsausgaben bremsend auswirkt, führt bei entsprechender demographischer Bildungsnachfrage zu hohen Privatausgaben. Besonderer Bedarf nach weiterer Forschung ist bei der näheren Erkundung des positiven Zusammenhangs zwischen der Frauenerwerbsquote und den öffentlichen, privaten und tertiären Bildungsausgaben anzumelden.

\section{Literatur}

Allmendinger, JuttalAisenbrey, Silke, 2002: Soziologische Bildungsforschung, in: Tippelt, Rudolf (Hrsg.), Handbuch Bildungsforschung. Opladen, 41-60.

Allmendinger, Jutta/Leibfried, Stephan, 2003: Education and the Welfare State: Germany's Poverty and Plenty and the Many Worlds of „competence distribution“ in the EC and the OECD, in: Journal of European Social Policy 13, 63-81.

Ansell, Ben W., 2006: From the Ballot to the Blackboard: Partisan and Institutional Effects on Education Policy. Paper presented at the 64th Annual Conference of the Midwest Political Science Association. Chicago, Ill., April 2006.

Beck, Nathaniel/Katz, Jonathan N., 1995: What to Do (and Not to Do) with Time-Series CrossSection Data, in: American Political Science Review 89, 634-647.

Beck, Nathaniel/Katz, Jonathan N., 1996: Nuisance vs. Substance: Specifiying and Estimating TimeSeries-Cross-Section Models, in: Political Analysis 6, 1-36.

Boix, Carles, 1997: Political Parties and the Supply Side of the Economy: The Provision of Physical and Human Capital in Advanced Economies, 1960-1990, in: American Journal of Political Science 41, 814-845.

Boix, Carles, 1998: Political Parties, Growth and Equality: Conservative and Social Democratic Economic Strategies in the World Economy. Cambridge.

Braun, Dietmar, 2000: The Territorial Division of Power in Comparative Public Policy Research: An Assessment, in: Braun, Dietmar (Hrsg.), Public Policy and Federalism. Aldershot, 27-56.

Büttner, Thiess/Schwager, Robert, 2004: Regionale Verteilungseffekte der Hochschulfinanzierung und ihrer Konsequenzen, in: Franz, Wolfgang/Ramser, Hans Jürgen/Stadler, Manfred (Hrsg.), Bildung, Wirtschaftswissenschaftliches Seminar Ottobeuren, 251-278.

Cameron, David R., 1978: The Expansion of the Public Economy: A Comparative Analysis, in: American Political Science Review 72, 1243-1261.

Cameron, David R., 1984: Social Democracy, Corporatism, Labor Quiescence and the Representation of Economic Interest in Advanced Capitalist Society, in: Goldthorpe, John H. (Hrsg.), Order and Conflict in Contemporary Capitalism. Oxford, 143-178.

Cameron, David R., 1985: Does Government Cause Inflation? Taxes, Spending, and Deficits, in: Lindberg, Leon N./Maier, Charles S./Barry, Brian M. (Hrsg.), The Politics of Inflation and Economic Stagnation. Washington, D.C., 224-279.

Cameron, David R./Hofferbert, Richard I., 1974: The Impact of Federalism on Education Finance: A Comparative Analysis, in: European Journal of Political Research 2, 225-258.

Castles, Francis G., 1982: The Impact of Parties on Public Expenditure, in: Castles, Francis G. (Hrsg.), The Impact of Parties: Politics and Policies in Democratic Capitalist States. London, 21-96.

Castles, Francis G., 1989: Explaining Public Education Expenditure in OECD Nations, in: European Journal of Political Research 17, 431-448.

Castles, Francis G., 1998: Comparative Public Policy: Patterns of Post-War Transformation. Cheltenham/Northampton, MA. 
Castles, Francis G., 2002: Policy Performance in the Democratic State: An Emergent Field of Study, in: Keman, Hans (Hrsg.), Comparative Democratic Politics: A Guide to Contemtporary Theory and Research. London et al., 215-232.

Castles, Francis G./Marceau, Jane, 1989: The Transformation in Gender Inequality in Tertiary Education, in: Journal of Public Policy 9, 493-507.

Castles, Francis G./McKinlay, Robert D., 1979: Does Politics Matter: An Analysis of the Public Welfare Commitment in Advanced Democratic States, in: European Journal of Political Research 7, 169-186.

Cohen-Zada, Danny/Justman, Moshe, 2003: The Political Economy of School Choice: Linking Theory and Evidence, in: Journal of Urban Economics 54, 277-308.

Czada, Roland, 2003: Der Begriff der Verhandlungsdemokratie und die vergleichende Policy-Forschung, in: Mayntz, Renate/Streeck, Wolfgang (Hrsg.), Die Reformierbarkeit der Demokratie: Innovationen und Blockaden. Festschrift für Fritz W. Scharpf. Frankfurt a.M., 173-204.

Esping-Andersen, Gosta, 1985: Power and Distributional Regimes, in: Politics \& Society 14, 223 256.

Esping-Andersen, Gosta, 1990: The Three Worlds of Welfare Capitalism. Cambridge.

Färber, Gisela, 2000: Bildungsreform durch Reform der Bildungsfinanzierung?, in: Weizsäcker, Robert (Hrsg.), Schul- und Hochschulorganisation. Berlin, 165-220.

Fernández, Raquel/Rogerson, Richard, 1997: The Determinants of Public Education Expenditures: Evidence from the States, 1950-1990. NBER Working Paper 5995. Cambridge, MA.

Garrett, Geoffrey, 2001: Globalization and Government Spending around the World, in: Studies in Comparative International Development 35, 3-29.

Garrett, Geoffrey/Lange, Peter, 1991: Political Responses to Interdependence: What's „Left" for the Left?, in: International Organization 45, 539-564.

Glennerster, Howard, 2001: United Kingdom Education 1997-2001. CASEpaper 50, Centre for Analysis of Social Exclusion, London School of Economics and Political Science. London.

Hanushek, Eric A./Rivkin, Steven G., 1996: Understanding the 20th Century Growth in U.S. School Spending. NBER Working Paper 5547. Cambridge, MA.

Heclo, Hugh, 1988: Generational Politics, in: Palmer, John L./Smeeding, Timothy/Torrey, Barbara Boyle (Hrsg.), The Vulnerable. Washington, DC, 381-411.

Hega, Gunther M./Hokenmaier, Karl G., 2002: The Welfare State and Education: A Comparison of Social and Educational Policy in Advanced Industrial Societies, in: German Policy Studies 2, $143-173$.

Hetmeier, Heinz-Werner/Weiß, Manfred, 2001: Bildungsausgaben, in: Böttcher, Wolfgang/Klemm, Klaus/Rauschenbach, Thomas (Hrsg.), Bildung und Soziales in Zahlen. Weinheim/München, $39-55$.

Heynemann, Stephen P., 2001: General Introduction: Global Issues in Education, in: Peabody Journal of Education 76, 1-6.

Hibbs, Douglas A., 1977: Political Parties and Macroeconomic Policy, in: American Political Science Review 71, 1467-1487.

Hibbs, Douglas A., 1987: The Political Economy of Industrial Democracies. Cambridge, MA/London.

Hicks, Alexander M./Swank, Duane H., 1992: Politics, Institutions, and Welfare Spending in Industrialized Democracies, 1960-1982, in: American Political Science Review 86, 658-674.

Hokenmaier, Karl G., 2002. Education, Social Security, and the Welfare State: Alternative Policy Choices in the United States and Germany. Kalamazoo.

Huber, Evelyne/Ragin, Charles/Stephens, John D., 1993: Social Democracy, Christian Democracy, Constitutional Structure, and the Welfare State, in: American Journal of Sociology 99, 711740 .

Huber, Evelyne/Stephens, John D., 2001a: Development and Crisis of the Welfare State: Parties and Policies in Global Markets. Chicago/London.

Huber, Evelyne/Stephens, John D., 2001b: Welfare State and Production Regimes in the Era of Retrenchment, in: Pierson, Paul (Hrsg.), The New Politics of the Welfare State. Oxford/New York, 107-145. 
Kaufman, Robert R./Segura-Ubiergo, Alex, 2001: Globalization, Domestic Politics, and Social Spending in Latin America: A Time-Series Cross-Section Analysis, 1973-97, in: World Politics 53, 553-587.

Kielmansegg, Peter Graf, 2001: Können Demokratien zukunftsverantwortlich handeln? Manuskript der Rede anlässlich der Verleihung des Schader-Preises 2001.

Kirchgässner, Gebhard, 2001: The Effects of Fiscal Institutions on Public Finance: A Survey of the Empirical Evidence. CESifo Working Paper Series No. 617, Discussion Paper No. 2001-15. St. Gallen.

Kirchgässner, Gebhard/Pommerehne, Werner W., 1997: Public Spending in Federal States: A Comparative Econometric Study, in: Capros, Pantélis/Meulders, Danièle (Hrsg.), Budgetary Policy Modelling: Public Expenditures. London/New York, 179-213.

Kittel, Bernhard/Obinger, Herbert, 2003: Political Parties, Institutions, and the Dynamics of Social Expenditure in Times of Austerity, in: Journal of European Public Policy 10, 20-45.

Kittel, Bernhard/Winner, Hannes, 2002: How Reliable is Pooled Analysis in Political Economy? The Globalization-Welfare State Nexus Revisited. MPIfG Discussion Paper 02/3. Köln.

Klemm, Klaus, 2003: Bildungsausgaben: Woher sie kommen, wohin sie fließen, in: Cortina, Kai S. (Hrsg.), Das Bildungswesen in der Bundesrepublik Deutschland. Reinbeck, 214-251.

Kohl, Jürgen, 1981: Trends and Problems in Postwar Public Expenditure Development in Western Europe and North America, in: Flora, Peter/Heidenheimer, Arnold J. (Hrsg.), The Development of Welfare States in Europe and America. New Brunswick/London, 307-344.

Landon, Stuart, 1999: Education Costs and Institutional Structure, in: Economics of Education Review 18: 327-345.

Leibfried, Stephan, 2003: Umspecken? Bildungspolitik im Sozialstaats-Umbau, in: Erziehung und Wissenschaft 6,12

Lepenies, Wolf, 2003: Das Ende der Bildungsrepublik, in: Süddeutsche Zeitung vom 6. und 7. Dezember, 4

Maddison, Agnus (Hrsg.), 2003: The World Economy. Historical Statistics. Paris.

Meyer, John W./Ramirez, Francisco O./Soysal, Yasemin Nuhoglu, 1992: World Expansion of Mass Education, 1870-1980, in: Sociology of Education 65, 128-149.

Morgan, David R./Kickham, Kenneth/LaPlant, James T., 2001: State Support for Higher Education: A Political Economy Approach, in: Policy Studies Journal 29, 359-371.

Nelson, F. Howard, 1992: The Myth of High Public Spending on American Education. American Federation of Teachers. Washington, DC.

Nelson, F. Howard, 1996: How and How Much the U.S. Spends on K-12 Education: An International Comparison. American Federation of Teachers. Washington, DC.

Obinger, Herbert/Wagschal, Uwe, 2000: Ökonomie, Institutionen und Politik: Determinanten der gebremsten Sozialstaatlichkeit im Überblick, in: Obinger, Herbert/Wagschal, Uwe (Hrsg.), Der gezügelte Wohlfahrtsstaat: Sozialpolitik in reichen Industrienationen. Frankfurt a.M./New York.

$O E C D$, 1992: Public Educational Expenditure, Costs and Financing. Paris.

$O E C D$, 2005: Education at a Glance: OECD Indicators. Paris.

$O E C D$, verschiedene Jahrgänge: Education at a Glance: OECD Indicators. Paris.

$O E C D$, verschiedene Jahrgänge: Labour Force Statistics. Paris.

Offe, Claus, 1973: Strukturprobleme des kapitalistischen Staates: Aufsätze zur Politischen Soziologie. Frankfurt a.M.

O'Higgins, Michael, 1988: The Allocation of Public Resources to Children and the Elderly in OECD Countries, in: Palmer, John L./Smeeding, Timothy/Torrey, Barbara Boyle (Hrsg.), The Vulnerable. Washington, DC., 201-228.

Olson, Mancur, 1982: The Rise and Decline of Nations: Economic Growth, Stagflation, and Social Rigidities. New Haven/London.

Olson, Mancur, 1992: Die Logik des kollektiven Handelns: Kollektivgüter und die Theorie der Gruppen. Tübingen.

Pampel, Fred C./Williamson, John B., 1988: Welfare Spending in Advanced Industrial Democracies, 1950-1980, in: American Journal of Sociology 93, 1424-1456. 
Pampel, Fred C./Williamson, John B., 1989: Age, Class, Politics, and the Welfare State. Cambridge et al.

Pecchenino, Rowena A./Utendorf, Kelvin R., 1999: Social Security, Social Welfare and the Aging Population, in: Journal of Population Economics 12, 607-623.

Pierson, Paul, 1994: Dismantling the Welfare State? Reagan, Thatcher and the Politics of Retrenchment. Cambridge.

Pierson, Paul, 1996: The New Politics of the Welfare State, in: World Politics 48, 143-179.

Pierson, Paul, 2001: Coping with Permanent Austerity: Welfare State Restructuring in Affluent Democracies, in: Pierson, Paul (Hrsg.), The New Politics of the Welfare State. Oxford, 410456.

Poterba, James M., 1997: Demographic Structure and the Political Economy of Public Education. Journal of Policy Analysis and Management 16, 48-66.

Ram, Rati, 1995: Public Educational Expenditures in the United States: An Analytical Comparison with Other Industrialized Countries, in: Economics of Education Review 14, 53-61.

Reuter, Lutz R., 2002: Politik- und rechtswissenschaftliche Bildungsforschung, in: Tippelt, Rudolf (Hrsg.), Handbuch Bildungsforschung. Opladen, 169-181.

Rodrik, Dani, 1997: Has Globalization Gone Too Far? Institute for International Economics. Washington, DC.

Rose, Richard, 1990: Inheritance before Choice in Public Policy, in: Journal of Theoretical Politics 2, 263-291.

Rose, RichardiDavies, Phillip L., 1994: Inheritance in Public Policy: Change without Choice in Britain. New Haven/London.

Santerre, Rexford E., 1989: Representative versus Direct Democracy: Are there any Expenditure Differences?, in: Public Choice 60, 145-154.

Sass, Tim R., 1991: The Choice of Municipal Government Structure and Public Expenditures, in: Public Choice 71, 71-87.

Schmidt, Manfred G., 1982: Wohlfahrtsstaatliche Politik unter bürgerlichen und sozialdemokratischen Regierungen: Ein internationaler Vergleich. Frankfurt a.M./New York.

Schmidt, Manfred G., 1993: Theorien der international vergleichenden Staatstätigkeitsforschung, in: Héritier, Adrienne (Hrsg.), Policy-Analyse: Kritik und Neuorientierung. PVS Sonderheft 24. Opladen, 371-393.

Schmidt, Manfred G., 1998: Sozialpolitik in Deutschland: Historische Entwicklung und internationaler Vergleich. Opladen.

Schmidt, Manfred G., 2000: Demokratietheorien. Opladen.

Schmidt, Manfred G., 2001: Einleitung, in: Schmidt, Manfred G. (Hrsg.), Wohlfahrtsstaatliche Politik: Institutionen, politischer Prozess und Leistungsprofil. Opladen, 7-29.

Schmidt, Manfred G., 2002a: The Impact of Political Parties, Constitutional Structures and Veto Players on Public Policy, in: Keman, Hans (Hrsg.), Comparative Democratic Politics: A Guide to Contemporary Theory and Research. London et al., 166-184.

Schmidt, Manfred G., 2002b: Warum Mittelmaß? Deutschlands Bildungsausgaben im internationalen Vergleich, in: Politische Vierteljahresschrift 43, 3-19.

Schmidt, Manfred G., 2003a: Ausgaben für Bildung im internationalen Vergleich, in: Aus Politik und Zeitgeschichte B 21/22, 6-11.

Schmidt, Manfred G., 2003b: Die parteipolitische Zusammensetzung von Regierungen der OECDDemokratien. Institut für Politische Wissenschaft, Universität Heidelberg.

Schmidt, Manfred G., 2004: Die öffentlichen und privaten Bildungsausgaben Deutschlands im internationalen Vergleich. ZSE 1, 7-31.

Siaroff, Alan, 1999: Corporatism in 24 Industrial Democracies: Meaning and Measurement, in: European Journal of Political Research 36, 175-205.

Siegel, Nico, 2002: Baustelle Sozialpolitik: Konsolidierung und Rückbau im internationalen Vergleich. Frankfurt a.M.

Stegarescu, Dan, 2004: Public Sector Decentralization: Measurement Concepts and Recent International Trends. ZEW Discussion Paper 04-74. Mannheim. 
Stephens, John D./Huber, Evelyne/Ray, Leonard, 1999: The Welfare State in Hard Times, in: Kitschelt, Herbert/Lange, Peter/Marks, Gary/Stephens, John D. (Hrsg.), Continuity and Change in Contemporary Capitalism. Cambridge, 164-193.

Timmermann, Dieter, 2002: Bildungsökonomie, in: Tipppelt, Rudolf(Hrsg.), Handbuch Bildungsforschung. Opladen, 81-122.

UNESCO, verschiedene Jahrgänge: Statistical Yearbook. Paris.

Verner, Joel G., 1979: Socioeconomic Environment, Political System, and Educational Policy Outcomes: A Comparative Analysis of 102 Countries, in: Comparative Politics 11, 165-187.

Weiß, Manfred, 1999: Bildungsfinanzierung im internationalen Vergleich, in: Rosenbladt, Bernhard von (Hrsg.), Bildung in der Wissensgesellschaft. Münster, 151-163.

Wildavsky, Aaron, 1964: The Politics of the Budgetary Process. Boston/Toronto.

Wilensky, Harold L., 1975: The Welfare State and Equality: Structural and Ideological Roots of Public Expenditures. Berkeley et al.

Wilensky, Harold L., 2002: Rich Democracies: Political Economy, Public Policy, and Performance. Berkeley et al.

Zöllner, Detlef, 1963: Öffentliche Sozialleistungen und wirtschaftliche Entwicklung. Berlin. 